\title{
Neurotoxin-induced ER stress in mouse dopaminergic neurons involves downregulation of TRPC1 and inhibition of AKT/mTOR signaling
}

\author{
Senthil Selvaraj, ${ }^{1}$ Yuyang Sun, ${ }^{1}$ John A. Watt, ${ }^{2}$ Shouping Wang, ${ }^{3}$ Saobo Lei, ${ }^{3}$ \\ Lutz Birnbaumer, ${ }^{4}$ and Brij B Singh ${ }^{1}$
}

\begin{abstract}
1Department of Biochemistry and Molecular Biology, ${ }^{2}$ Department of Anatomy and Cell Biology, and ${ }^{3}$ Department of Physiology, Pharmacology and Therapeutics, School of Medicine and Health Sciences, University of North Dakota, Grand Forks, North Dakota, USA. ${ }^{4}$ Laboratory of Signal Transduction, National Institute of Environmental Health Sciences (NIEHS), NIH, Research Triangle Park, North Carolina, USA.
\end{abstract}

\begin{abstract}
Individuals with Parkinson's disease (PD) experience a progressive decline in motor function as a result of selective loss of dopaminergic (DA) neurons in the substantia nigra. The mechanism(s) underlying the loss of DA neurons is not known. Here, we show that a neurotoxin that causes a disease that mimics PD upon administration to mice, because it induces the selective loss of DA neurons in the substantia nigra, alters $\mathrm{Ca}^{2+}$ homeostasis and induces ER stress. In a human neuroblastoma cell line, we found that endogenous store-operated $\mathrm{Ca}^{2+}$ entry (SOCE), which is critical for maintaining $\mathrm{ER} \mathrm{Ca}^{2+}$ levels, is dependent on transient receptor potential channel 1 (TRPC1) activity. Neurotoxin treatment decreased TRPC1 expression, TRPC1 interaction with the SOCE modulator stromal interaction molecule 1 (STIM1), and $\mathrm{Ca}^{2+}$ entry into the cells. Overexpression of functional TRPC1 protected against neurotoxin-induced loss of SOCE, the associated decrease in ER $\mathrm{Ca}^{2+}$ levels, and the resultant unfolded protein response (UPR). In contrast, silencing of TRPC1 or STIM1 increased the UPR. Furthermore, $\mathrm{Ca}^{2+}$ entry via TRPC1 activated the AKT pathway, which has a known role in neuroprotection. Consistent with these in vitro data, $\operatorname{Trpc1} 1^{-/-}$mice had an increased UPR and a reduced number of DA neurons. Brain lysates of patients with PD also showed an increased UPR and decreased TRPC1 levels. Importantly, overexpression of TRPC1 in mice restored AKT/mTOR signaling and increased DA neuron survival following neurotoxin administration. Overall, these results suggest that TRPC1 is involved in regulating $\mathrm{Ca}^{2+}$ homeostasis and inhibiting the UPR and thus contributes to neuronal survival.
\end{abstract}

\section{Introduction}

Parkinson's disease (PD) is the second most common neurodegenerative disorder and is characterized by the selective loss of dopaminergic (DA) neurons in the substantia nigra pars compacta region $(\mathrm{SNpc})$. Loss of DA neurons leads to a decrease in motor function resulting in symptoms that include resting tremor, rigidity, bradykinesia, and postural instability $(1,2)$. Although the cause of PD is not known, recent research suggests that more than $90 \%$ of PD cases are of idiopathic origin (3). Likewise, the mechanisms leading to selective DA neuronal loss in SNpc are also not fully understood. In recent years, attention has turned to the role of $\mathrm{Ca}^{2+}$ in $\mathrm{PD}$, and it has been shown that L-type $\mathrm{Ca}^{2+}$ channels make DA neurons susceptible to mitochondrial toxins (4). Furthermore, changes in $\mathrm{Ca}^{2+}$ homeostasis especially in storage organelles, ER, and mitochondria have been shown to affect neuronal survival and are closely linked with PD (5). ER is a large organelle that serves as storage for $\mathrm{Ca}^{2+}$ ions, which is necessary for regulating protein translation, membrane folding, and protein secretion (6). Impairment of $\mathrm{ER} \mathrm{Ca}^{2+}$ homeostasis, including ER $\mathrm{Ca}^{2+}$ depletion or inhibition of $\mathrm{N}$-linked glycosylation, leads to the accumulation of unfolded/misfolded proteins in the ER lumen, thereby causing ER stress (7). As a defense mechanism, cells activate the unfolded protein response (UPR), thereby increasing ER

Conflict of interest: The authors have declared that no conflict of interest exists. Citation for this article: JClin Invest. 2012;122(4):1354-1367. doi:10.1172/JCI61332. chaperones and activating an ER-associated degradation (ERAD) pathway that is necessary to alleviate ER stress and improve cell survival $(8,9)$. However, prolonged activation of the UPR due to severe ER dysfunction results in programmed cell death (10).

The neurotoxin 1-methyl-4-phenyl-1,2,3,6-tetrahydropyridine (MPTP) has been used to develop PD models, as it induces selective loss of DA neurons in the SNpc. Systemically administered MPTP crosses the blood brain barrier and is taken up by glial cells, where it is metabolized/oxidized to 1-methyl-4-phenylpyridinium $\left(\mathrm{MPP}^{+}\right) . \mathrm{MPP}^{+}$is then released and is specifically taken up by DA neurons via dopamine transporters and inhibits mitochondrial complex I activity (11-13). The cellular consequences of mitochondrial dysfunction, as induced by $\mathrm{MPP}^{+}$, are numerous and include disturbance in $\mathrm{Ca}^{2+}$ homeostasis and oxidative stress (14, $15)$. Results from various PD models and analysis of postmortem PD samples also point toward a role for ER stress in PD pathogenesis $(16,17)$. However, although it is apparent that ER stress plays a major role in neurodegeneration, the mechanism by which these neurotoxins induce ER stress is not known. Previously we reported that transient receptor potential channel 1 (TRPC1) is critical for neuronal survival and that $\mathrm{MPP}^{+}$treatment decreases TRPC1 expression in SH-SY5Y and PC12 cells $(18,19)$; however, the mechanism is not known.

Members of the TRPC family have been suggested as mediators of $\mathrm{Ca}^{2+}$ entry into cells (20-22). Activation of the $\mathrm{G}$ protein $\left(\mathrm{G}_{\mathrm{q} / 11}\right)$ / PLC signaling pathway leads to phosphatidylinositol 4,5-bisphos- 
phate $\left(\mathrm{PIP}_{2}\right)$ hydrolysis that generates inositol trisphosphate $\left(\mathrm{IP}_{3}\right)$ and diacylglycerol (DAG) (23). IP 3 binds to the $\mathrm{IP}_{3}$ receptor $\left(\mathrm{IP}_{3} \mathrm{R}\right)$ and initiates $\mathrm{Ca}^{2+}$ release from the ER stores, which allows stromal interacting molecule 1 (STIM1) to rearrange and activate $\mathrm{Ca}^{2+}$ entry (24). $\mathrm{Ca}^{2+}$ entry through store-operated channels (SOCs) is essential for the refilling of $\mathrm{ER} \mathrm{Ca}^{2+}$ stores as well as in regulating cellular functions. Two families of proteins (TRPCs and Orais) have been identified as potential candidates for SOC-mediated $\mathrm{Ca}^{2+}$ entry $(21,24-29)$. However, their role in PD has not yet been determined. Thus, this study aimed to elucidate the mechanism of $\mathrm{MPTP} / \mathrm{MPP}^{+}$-mediated loss of DA neurons and to identify key molecular players that regulate neuronal survival. We report for the first time to our knowledge that the endogenous SOC channel in DA neurons is dependent on TRPC1 and that MPTP/MPP+ induced loss of TRPC1 function induces ER stress. Furthermore, activation of TRPC1 initiates $\mathrm{Ca}^{2+}$ entry that regulates the AKT/ mTOR pathway, which is essential for the protection of DA neurons against neurotoxins that induce PD-like symptoms.

\section{Results}

Evidence that loss of ER Ca ${ }^{2+}$ induces ER stress in cultured cells and that ER stress is increased in PD and in neurotoxin-induced animal models that mimic $P D$. Previous studies have suggested that the unfolded protein response (UPR) could be one of the reasons for the loss of DA neurons (17); however, the mechanism that triggers the UPR is not known. Thus, we examined this mechanism by evaluating the status of UPR proteins, critical for initiating ER stress in in vivo and in vitro PD models. As shown in Figure 1, UPR markers (GRP78 and CHOP) were upregulated at both the mRNA (Figure $1 \mathrm{~B}$ ) and the protein levels (Figure 1A) in the SNpc region of postmortem brains from PD patients when compared with agematched control samples (quantification shown in Supplemental Figure 1A; supplemental material available online with this article; doi:10.1172/JCI61332DS1). Based on these findings, we assessed whether neurotoxin-induced experimental PD models show signs of an activated UPR. As shown in Figure 1C, GRP78 and CHOP were also increased in the SNpc of mice treated with MPTP. Moreover, addition of $\mathrm{MPP}^{+}$to SH-SY5Y neuroblastoma cells significantly increased the expression of ER chaperones GRP78/Bip and GRP94 (Figure 1D). Importantly, increased expression of both GRP78 and GRP94 was observed after 3 hours MPP treatment and remained elevated for 12 hours (Figure 1D). In addition, $\mathrm{CHOP}$, which is an important mediator of ER stress-induced apoptosis, was upregulated at 6 hours of $\mathrm{MPP}^{+}$treatment (Figure 1D). Quantification of individual proteins showed a $60 \%$ increase in their expression after 12 hours of $\mathrm{MPP}^{+}$treatment when compared with control cells (Figure 1E), indicating that $\mathrm{MPP}^{+}$activates a persistent UPR in SH-SY5Y cells. To confirm these results, we performed luciferase assays to evaluate the activation of the ER stress response element (ERSE), which is present in the promoter region of various UPR target genes, including CHOP. As shown in Figure 1F, a time-dependent increase in ERSE activity was observed after $\mathrm{MPP}^{+}$treatment, further suggesting that addition of $\mathrm{MPP}^{+}$induces ER stress. Overall, the results obtained from PD patients and experimental models of PD clearly revealed that ER stress is activated in PD and could lead to neurodegeneration.

To determine the mechanism(s) underlying $\mathrm{MPP}^{+}$-induced ER stress, we investigated the effect of $\mathrm{MPP}^{+}$on SOC-mediated $\mathrm{Ca}^{2+}$ entry, since SOC-mediated $\mathrm{Ca}^{2+}$ entry is essential for maintaining $\mathrm{ER} \mathrm{Ca}^{2+}$ levels and loss of $\mathrm{ER} \mathrm{Ca}^{2+}$ can initiate UPR. For evaluation of SOC-mediated $\mathrm{Ca}^{2+}$ entry, ER $\mathrm{Ca}^{2+}$ stores were depleted by the addition of thapsigargin ( $\mathrm{Tg}, 2 \mu \mathrm{M})$, a sarcoplasmic/endoplasmic reticulum $\mathrm{Ca}^{2+}$ ATPase (SERCA) pump blocker. Importantly, in the absence of extracellular $\mathrm{Ca}^{2+}$, the increase in intracellular $\mathrm{Ca}^{2+}$ $\left(\left[\mathrm{Ca}^{2+}\right]_{i}\right)$ evoked by Tg (first peak) was significantly decreased following 3 hours of $\mathrm{MPP}^{+}$treatment, when compared with control untreated cells (Figure 1, G and H). Subsequently, addition of external $\mathrm{Ca}^{2+}(1 \mathrm{mM})$, which initiates SOC-mediated $\mathrm{Ca}^{2+}$ entry, was decreased even within 1 hour of $\mathrm{MPP}^{+}$treatment. Together these results suggest that loss of SOC-mediated $\mathrm{Ca}^{2+}$ entry could decrease $\mathrm{ER} \mathrm{Ca}^{2+}$ levels and initiate the UPR response. To establish the identity of the SOC channel, we performed electrophysiological recordings. Addition of Tg induced an inward current that was nonselective and reversed between 0 and $-5 \mathrm{mV}$ (Figure 1, I-K; characterization of the currents shown in Supplemental Figure $1, \mathrm{~B}-\mathrm{I})$. The currents shown are recorded at a holding potential of $-80 \mathrm{mV}$, and maximum peak currents were used for tabulation. The current-voltage (I-V) curves were made using a ramp protocol wherein current density was evaluated at various membrane potentials and plotted in the figure. Importantly, the channel properties were similar to those previously observed with TRPC1 channels and the activity was blocked by $\mathrm{Gd}^{3+}(26)$, suggesting that TRPC1 could contribute to the endogenous SOC-mediated $\mathrm{Ca}^{2+}$ entry channel in SH-SY5Y cells. Also, SKF-96365, a nonspecific TRPC channel blocker, decreased these inward currents in SH-SY5Y cells (Figure 1K and Supplemental Figure 1, H and I). Importantly, the $\mathrm{MPP}^{+}$treatment significantly decreased SOC currents without altering the I-V relationship (Figure 1J). Similar results were also obtained in differentiated SH-SY5Y cells (retinoic acid treatment), where $\mathrm{MPP}^{+}$treatment decreased SOC-mediated $\mathrm{Ca}^{2+}$ entry (Supplemental Figure 1J). Collectively, these results suggest that $\mathrm{MPP}^{+}$decreases ER $\mathrm{Ca}^{2+}$ by diminishing SOC-mediated $\mathrm{Ca}^{2+}$ entry, which could lead to the activation of the UPR in these cells. Importantly, although 1-hour treatment with $\mathrm{MPP}^{+}$or addition of $\mathrm{MPP}^{+}$in the patch pipette decreased SOC-mediated $\mathrm{Ca}^{2+}$ entry, no cell death was observed until 12 hours of treatment with $\mathrm{MPP}^{+}$(Supplemental Figure 1, K-M). Importantly, since ER $\mathrm{Ca}^{2+}$ was decreased after 3 hours and ER stress was induced after 6 hours of $\mathrm{MPP}^{+}$treatment, it can be hypothesized that the loss of SOC-mediated $\mathrm{Ca}^{2+}$ entry is the early event that could lead to ER stress followed by neurotoxin-induced neuronal loss.

$M P P^{+}$decreases SOC-mediated $\mathrm{Ca}^{2+}$ entry by reducing TRPC1 expression. Given the importance of $\mathrm{MPP}^{+}$-induced ER stress caused by the loss of $\mathrm{Ca}^{2+}$ homeostasis, we next studied the expression of SOC(s) that were affected by prolonged treatment with $\mathrm{MPP}^{+}$. Although the molecular component(s) of SOCs in neurons are not known, members of TRPC and Orai that have been shown as candidates of SOC channels in many cell types $(24,27)$ could be present in neuronal cells. To address this issue, we performed real-time RT-PCR analysis to evaluate changes in TRPC mRNA. As shown in Figure 2A, a significant decrease in expression of TRPC1, but not other TRPCs (TRPC3, TRPC5, and TRPC6), was observed in $\mathrm{MPP}^{+}$-treated cells. TRPC4 and TRPC7 were not expressed in these cells. Western blot analysis confirmed the loss of TRPC1 after $\mathrm{MPP}^{+}$treatment (12 hours), whereas no change in the expression of either Orai1 or STIM1 (a regulator for TRPC1 and Orai1) (24, 27) was observed (Figure 2, B and C).

Previous studies have shown that upon store depletion, STIM1 interacts with TRPC1 as well as with Orai1 and thereby initiates $\mathrm{Ca}^{2+}$ entry $(24,28)$. Thus, to further confirm that TRPC1 is critical 
A

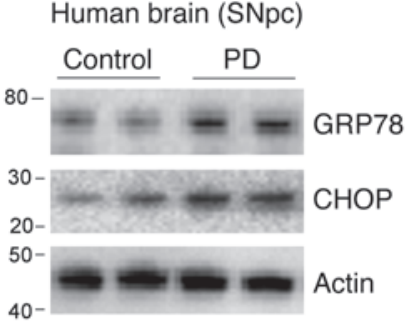

D

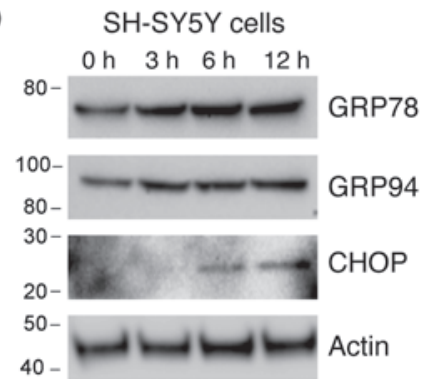

B

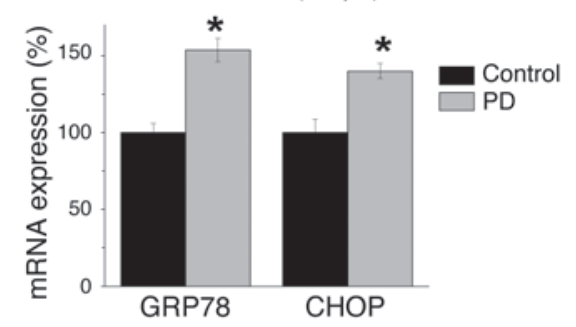

E

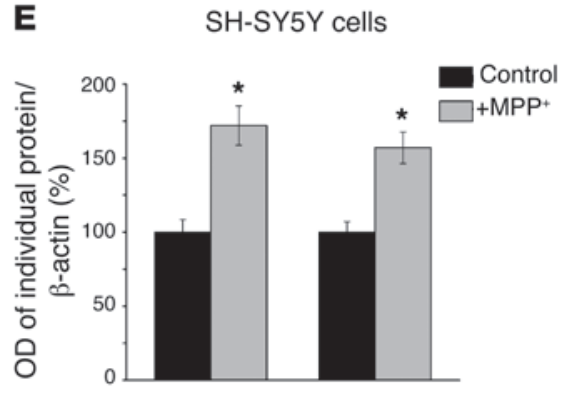

C
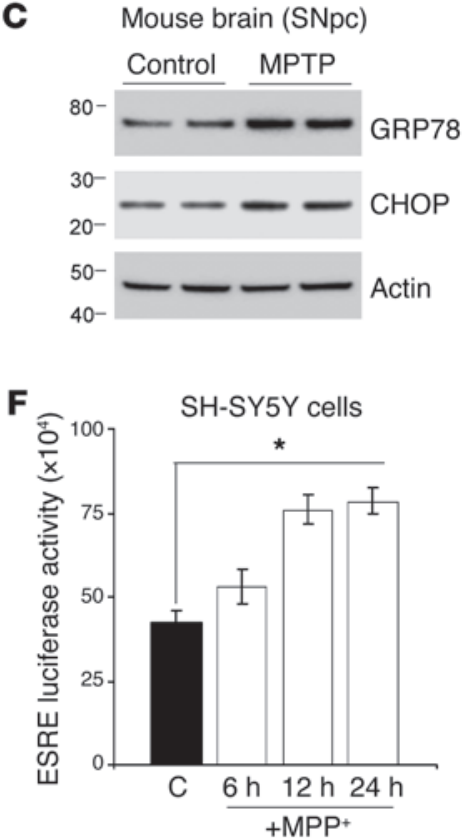

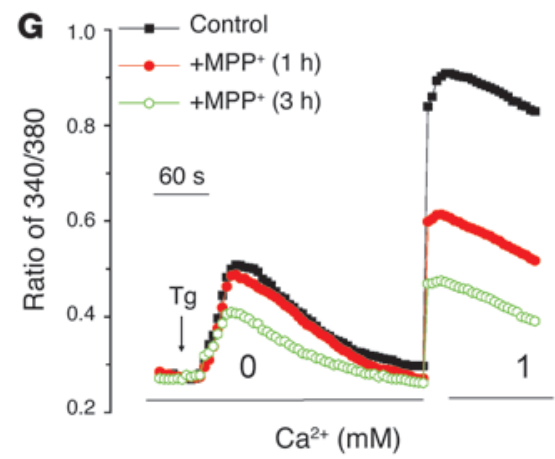

I

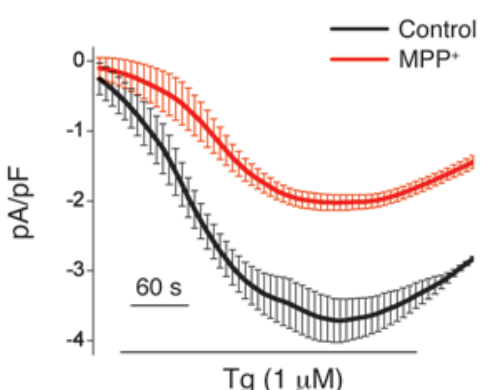

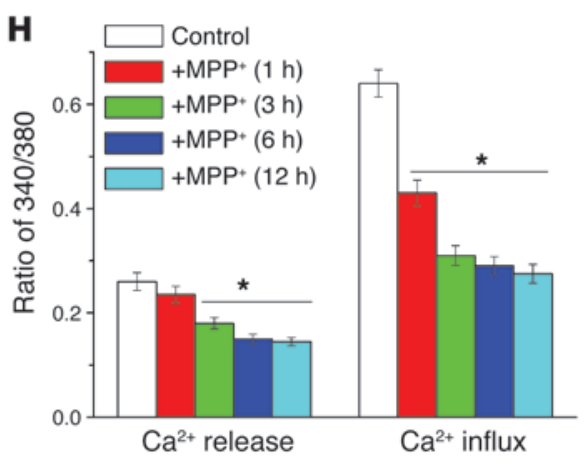

J

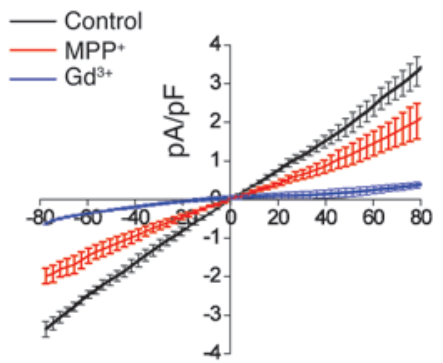

$\mathbf{K}$

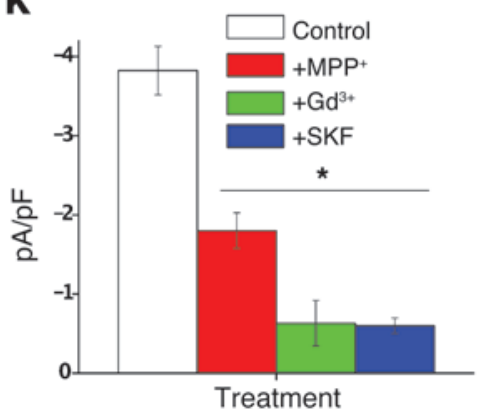

Figure 1

$\mathrm{MPP}+$ induces ER Ca${ }^{2+}$ depletion by attenuating SOCE, which causes ER stress. (A) Representative blots from the SNpc region of postmortem human PD $(n=5)$ and age-matched controls $(n=4)$. (B) RNA was extracted, and RT-PCR was performed. Values represent mean \pm SD from 3 independent experiments $\left({ }^{*} P<0.05\right)$. (C) Mice received $25 \mathrm{mg} / \mathrm{kg}$ MPTP as described in ref. 19, and SNpc samples were removed, processed, and immunoblotted using the respective antibodies. (D) SH-SY5Y cells were treated with $500 \mu \mathrm{M} \mathrm{MPP}+$, and cell lysates were resolved and analyzed by Western blotting. Antibodies are labeled; $\beta$-actin was used as loading control. (E) Quantification (mean \pm SD) from 3 or more independent experiments. The OD of GRP78 and GRP94 was normalized to $\beta$-actin. (F) Cells transfected with the ERSE promoter were lysed after $\mathrm{MPP}+$ treatment, and luciferase assays were performed. Values represent mean $\pm \mathrm{SD}$ from 3 independent experiments $\left({ }^{*} P<0.05\right)$. (G) Analog plots of the fluorescence ratio $(340 / 380 \mathrm{~nm}$ ) from an average of 30-40 cells in each condition. (H) Quantification (mean \pm SEM) of 340/380 ratio. ${ }^{*} P<0.05$ versus untreated. (I) Tg-induced currents (mean \pm SEM) were evaluated in control and MPP+-treated (12 hours) cells. The holding potential for current recordings was $-80 \mathrm{mV}$. (J) I-V curves (mean current \pm SEM) under these conditions; the average (8-10 recordings) current intensity under various conditions is shown in $\mathbf{K}$. ${ }^{*} P<0.05$ compared with control; values are shown as mean \pm SEM. SKF, SKF- 96365 . 
A

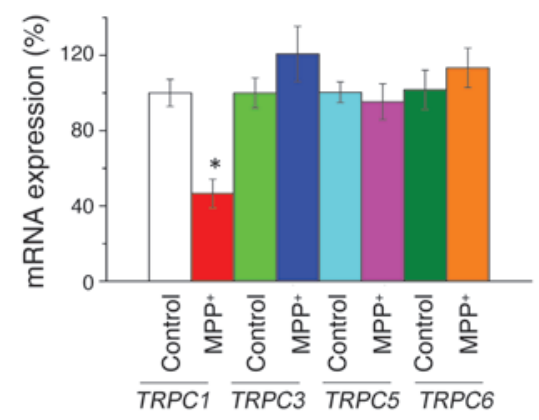

D

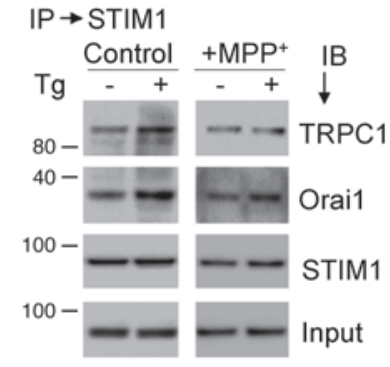

B

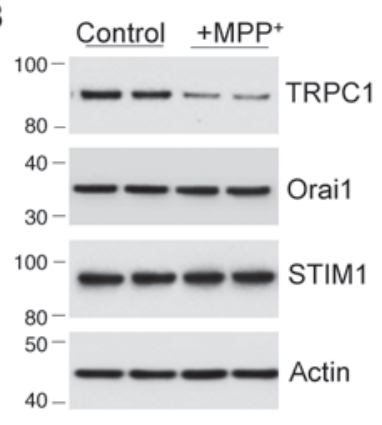

E

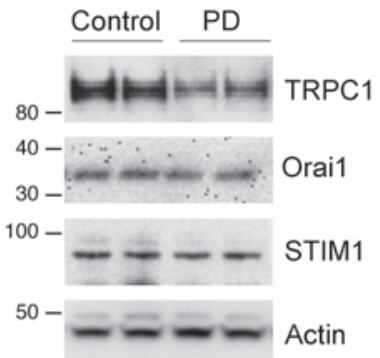

C

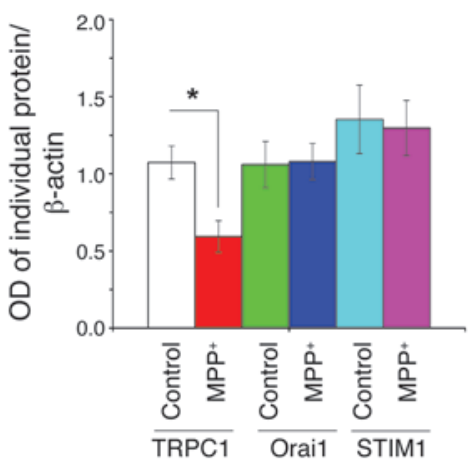

F

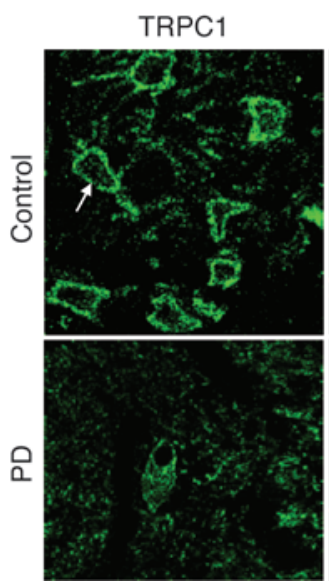

$\mathrm{TH}$

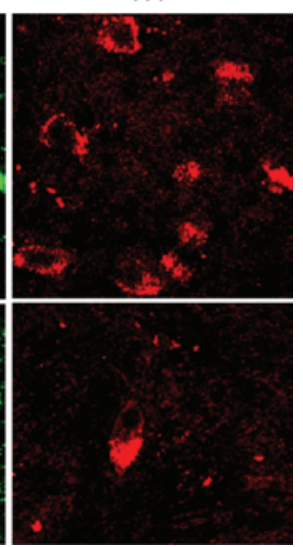

\section{Figure 2}

TRPC1 mediates SOCE in SH-SY5Y cells, and MPP+ selectively decreases TRPC1 expression/function. (A) SH-SY5Y cells were treated with $\mathrm{MPP}^{+}(500 \mu \mathrm{M})$ for 12 hours. RNA was extracted, and quantitative RT-PCR was performed. Values represent mean \pm SD from at least 3 independent experiments. ${ }^{*} P<0.05$ versus untreated control. TRPC1 was evaluated after 15 cycles, whereas other TRPCs required at least 25 cycles. (B) Cells were treated with vehicle control or MPP+ and lysed, and proteins were subjected to SDS-PAGE followed by Western blotting with the indicated antibodies. Membranes were reprobed with anti- $\beta$-actin antibody to confirm equal loading. (C) Quantification (mean \pm SD) of individual proteins from 3 or more independent experiments. Relative expression of each individual proteins was normalized to $\beta$-actin. ${ }^{\star} P<0.05$ versus untreated control. (D and E) Immunoprecipitation with anti-STIM1 antibody of lysates from control or MPP+-treated cells with or without Tg. Immunoblotting was performed using anti-TRPC1, -Orai1, and -STIM1 antibodies. (E) Brain lysates from control and PD samples were subjected to SDS-PAGE and immunoblotted with the respective antibodies. (F) Paraffin-embedded sections of postmortem human SNpc samples obtained from control and PD patients were immunostained using TRPC1 and TH antibodies. Original magnification, $\times 40$.

for $\mathrm{Ca}^{2+}$ entry in these cells, we performed co-immunoprecipitation experiments. Importantly, Tg-mediated store depletion induced STIM1-TRPC1 interaction in SH-SY5Y cells, which was decreased in $\mathrm{MPP}^{+}$-treated cells (Figure 2D). In addition, association of STIM1 with Orai11, which is also shown to increase upon store depletion (28), was unaffected upon $\mathrm{MPP}^{+}$treatment (Figure 2D; quantification provided in Supplemental Figure 2, A and B). Together these data suggest that TRPC1 is essential for store-operated $\mathrm{Ca}^{2+}$ entry (SOCE) in SH-SY5Y cells and that $\mathrm{MPP}^{+}$decreases SOCE by decreasing TRPC1 expression and TRPC1-STIM1 interaction. While the above results suggest the significance of TRPC1 in an in vitro PD model, nothing is known about its function in PD patients. Thus, we further explored the potential relevance of TRPC1 in PD by evaluating TRPC1 expression in the SNpc of control and PD patients. Expression of TRPC1, but not Orai1 or STIM1, was decreased in the SNpc of PD patients as compared with age-matched control $\mathrm{SNpc}$ tissues (Figure 2E; quantification is shown in Supplemental Figure 2C). Moreover, TRPC1 was localized in or near the plasma membrane of the DA neurons, and expression was decreased in PD patients (Figure 2F). Similar results were also obtained in mouse primary DA cells, which also showed a significant decrease in TRPC1 expression when treated with $\mathrm{MPP}^{+}$(Supplemental Figure $2 \mathrm{~F}$ ). Taken together, these findings suggest that PD patients have decreased TRPC1 expression in DA neurons; however, since these samples were obtained from patients at stages 3 and 4 of PD, caution should be used before interpreting these results, and more samples from patients at the early stage of disease are needed to confirm the loss of TRPC1 in PD samples.

Attenuation of SOC-mediated $\mathrm{Ca}^{2+}$ entry or deletion of TRPC1 induces ER stress. TRPC1 is ubiquitously expressed, including in the SNpc (30), and although TRPC1 allows plasma membrane $\mathrm{Ca}^{2+}$ influx in response to $\mathrm{ER} \mathrm{Ca}^{2+}$ depletion, so far there are no reports showing that TRPC1 mediates SOCE in SH-SY5Y cells. To address this, we silenced TRPC1 using TRPC1 siRNA and assessed both ER $\mathrm{Ca}^{2+}$ release and $\mathrm{Ca}^{2+}$ influx upon store depletion. Interestingly, Tg-induced SOC currents were significantly decreased in TRPC1 siRNA-transfected cells when compared with control siRNAtransfected cells (Figure 3, A and B). RNAi-mediated knockdown 

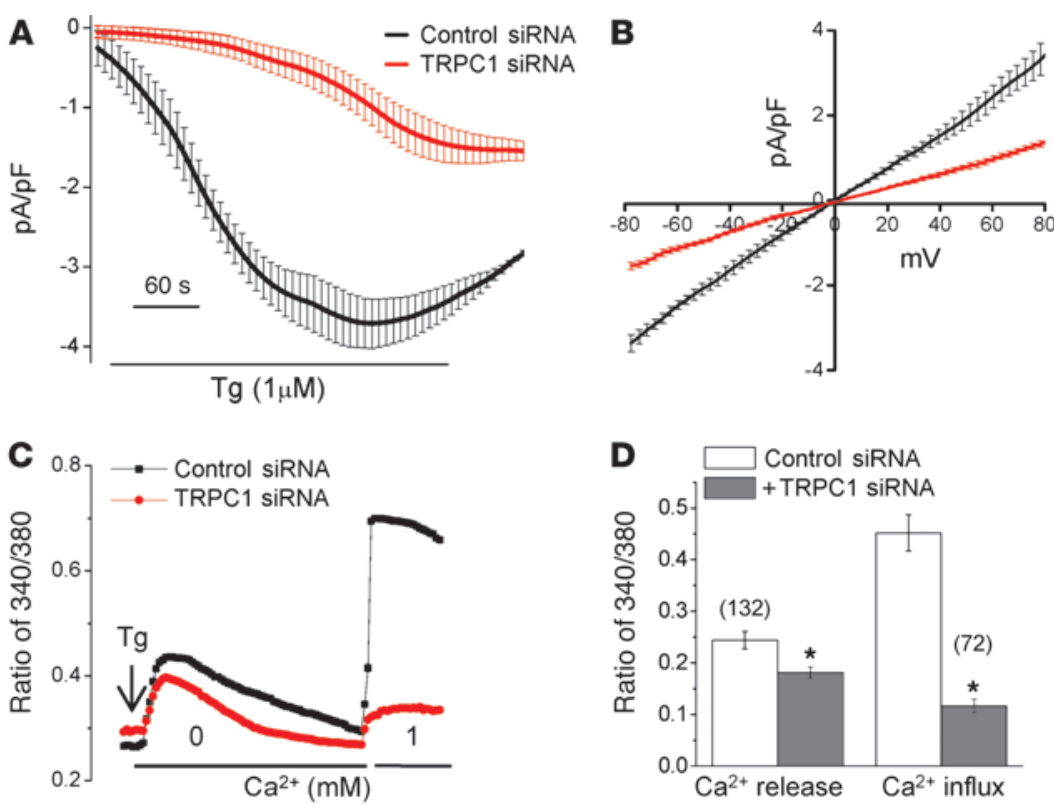

$\mathbf{F}$

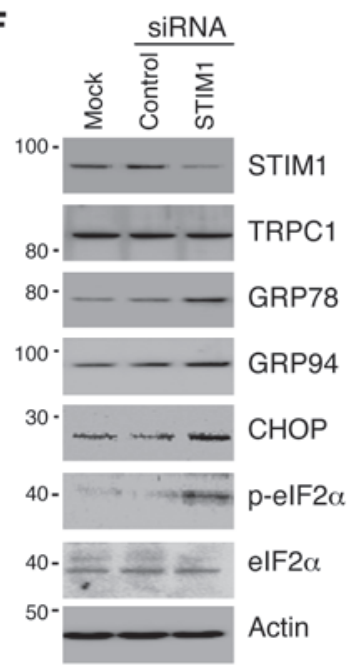

I Brain slice; holding $-70 \mathrm{mv}$

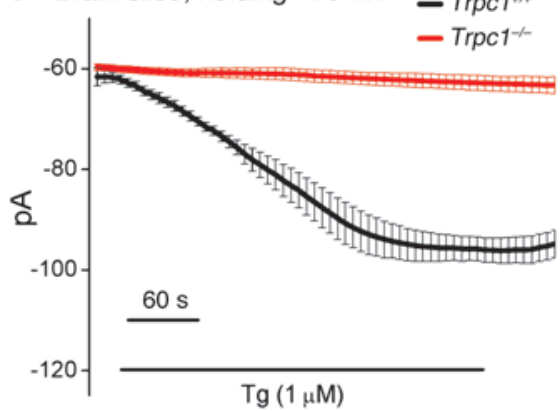

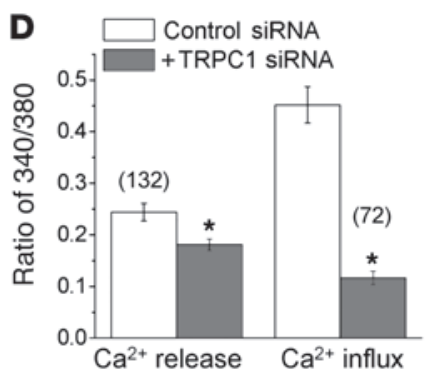

G

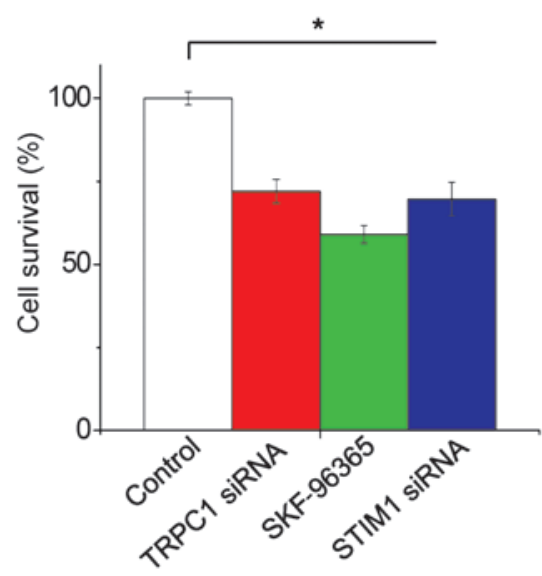

J

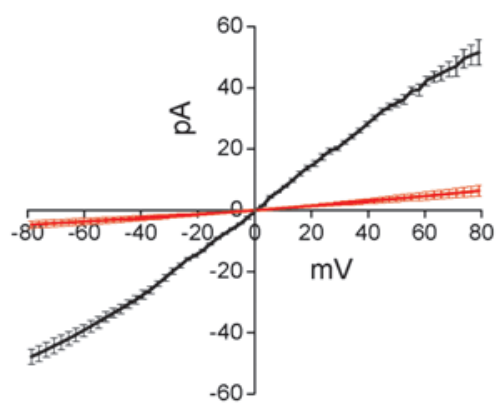

E

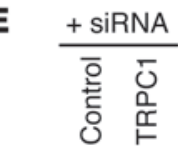

+ SKF $(\mu \mathrm{M})$

응 유 우

$80-10$

$100-1+1.0$.

$30-$

120 - - D-PERK

120 - - PERK

$40--p$-elF2 $\alpha$

$40--1-$ elF2o

$50-=$ Actin

\section{H}

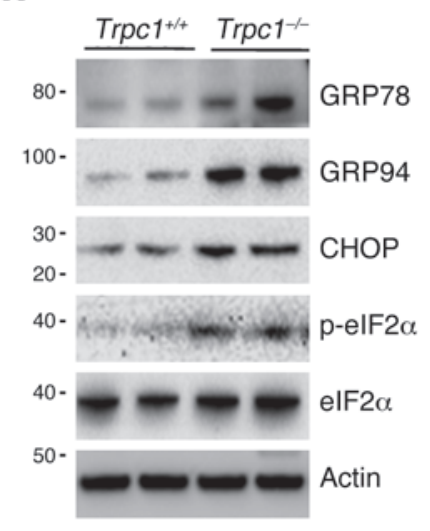

K

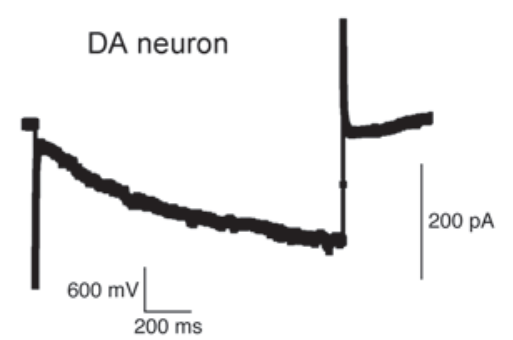




\section{Figure 3}

TRPC1 functions as an endogenous SOCE channel, and knockdown of TRPC1 induces ER stress. (A) Tg-induced currents (mean \pm SEM) were evaluated in control siRNA- and TRPC1 siRNA-transfected cells. The holding potential for the recordings was $-80 \mathrm{mV}$, and an $\mathrm{I}-\mathrm{V}$ curve (mean current \pm SEM) under these conditions is shown in B. (C) Analog plots of the $340 / 380$ ratio from an average of $40-60$ cells are shown. (D) Quantification (mean \pm SEM) of fluorescence ratio. ${ }^{\star} P<0.05$ versus untreated control; numbers of cells imaged are indicated. (E) SH-SY5Y cells were transfected with control siRNA or TRPC1 siRNA, or treated with 50 or $100 \mu \mathrm{M}$ SKF-96365 for 24 hours. Cells were lysed, subjected to SDS-PAGE, and immunoblotted with the respective antibodies. (F) SH-SY5Y cells transfected with control or STIM1 siRNA were lysed and immunoblotted with respective antibodies. (G) MTT assay was performed in control, TRPC1 siRNAtransfected, SKF-96365-treated (100 $\mu \mathrm{M}$ for 24 hours), or STIM1 siRNA-transfected cells. Values represent mean \pm SD from at least 3 independent experiments. ${ }^{*} P<0.05$ versus control. $(\mathbf{H})$ Tissue lysates from the SNpc region of wild-type and Trpc1-/- mice were subjected to SDS-PAGE and immunoblotted with the respective antibodies. (I and J) Endogenous currents (mean \pm SEM) and relative I-V curves (mean currents \pm SEM) upon Tg stimulation in DA neurons in the SNpc of $\mathrm{Trpc1}^{+/+}$and $\mathrm{Trpc}^{1 /-}$ mice. The currents shown were recorded at a holding potential of $-70 \mathrm{mV}$. (K) DA neurons induced a large inward current by a hyperpolarizing pulse of $60 \mathrm{mV}$, indicating the electrical signature of DA neurons.

of TRPC1 not only abolished $\mathrm{Ca}^{2+}$ entry activated by store depletion, but also led to a significant decrease in $\mathrm{ER} \mathrm{Ca}^{2+}$ (Figure 3, C and D). The efficiency of siRNA-mediated TRPC1 knockdown in SH-SY5Y cells was confirmed by Western blotting (Supplemental Figure 2D). These results suggested that TRPC1 is essential for SOC-mediated $\mathrm{Ca}^{2+}$ entry and that deletion of TRPC1 affects ER/ cytosolic $\mathrm{Ca}^{2+}$ homeostasis. We further studied whether blocking of TRPC1 function or silencing of TRPC1 induces ER stress in SHSY5Y cells. Indeed, TRPC1 silencing induced a UPR, which was clearly evidenced by increased expression of GRP78, GRP94, and CHOP (Figure 3E; quantification shown in Supplemental Figure 2E). Also, silencing of TRPC1 led to increased phosphorylation of PERK and its downstream effector eIF2 $\alpha$. Similarly, blocking TRPC1 channel activity with SKF-96365 led to an increase in the UPR and inhibited protein translation by increasing eIF $2 \alpha$ phosphorylation (Figure 3E; quantification shown in Supplemental Figure 3, A-G). Interestingly, silencing of TRPC3 failed to upregulate UPR (Supplemental Figure 3F). These results indicate that inhibition of SOC-mediated $\mathrm{Ca}^{2+}$ entry could be critical in inducing ER stress in SH-SY5Y cells.

To further validate this hypothesis, we repressed SOC-mediated $\mathrm{Ca}^{2+}$ entry by silencing STIM1, which again induced ER stress by increasing the expression of GRP78, GRP94, CHOP, and phosphoeIF2 $\alpha$ (Figure 3F; quantification shown in Supplemental Figure 4, A-C). Moreover, either silencing of TRPC1 or STIM1 or blocking of TRPC channel activity decreased the survival of SH-SY5Y cells (Figure 3G). Consistent with these results, $\operatorname{Trpc1}^{-/-}$mice had increased GRP78, GRP94, CHOP, and p-eIF2 $\alpha$ levels (Figure 3H and Supplemental Figure 4D) compared with wild-type $\left(\operatorname{Trpc1} 1^{+++}\right)$. To determine whether SOC channels are also present in native DA neurons, we performed electrophysiological recordings in DA neurons $\left(\mathrm{SNpc}\right.$ ) of $\operatorname{Tr} p c 1^{+/+}$and $\operatorname{Tr} p c 1^{-/-}$mice. Interestingly, addition of $\mathrm{Tg}$ in the SNpc of $\mathrm{Trpc}^{+/+}$initiated a linear, nonselective current in DA neurons, which was similar to the currents observed in SH-SY5Y cells and was absent in Trpc1-/- mice (Figure 3, I-K, and
Supplemental Figure 4E). The electrical signature present in DA neurons was used to confirm that indeed these are DA neurons (Figure 3K and Supplemental Figure 4F). Collectively, these results reveal that TRPC1 mediates SOC-mediated $\mathrm{Ca}^{2+}$ entry in DA cells/ neurons and that inhibition of $\mathrm{Ca}^{2+}$ entry (by TRPC1 or STIM1 silencing or blocking of TRPC1 channel activity) prevents optimal refilling of $\mathrm{ER} \mathrm{Ca}^{2+}$, thereby inducing ER stress.

Overexpression of TRPC1 restores SOC-mediated $\mathrm{Ca}^{2+}$ entry and attenuates ER stress. The results shown above suggest that TRPC1 could be critical for SOC-mediated $\mathrm{Ca}^{2+}$ entry and in maintaining $\mathrm{ER} \mathrm{Ca}^{2+}$ homeostasis; however, to confirm the role of TRPC1, we next overexpressed TRPC1 and evaluated its role in ER $\mathrm{Ca}^{2+}$ homeostasis and the ER stress response. SH-SY5Y cells were infected with Ad-HA-TRPC1 at an MOI of 5, and Ad-GFP (MOI of 5) was used as control. The efficiency of TRPC1 expression (HATRPC1) was confirmed by Western blotting (Supplemental Figure 5A). Importantly, overexpression of TRPC1, but not TRPC3 or Orai1, increased SOC currents (without changing the I-V relationship) and led to increased cell survival (Figure 4, A and B, and Supplemental Figure 5, B and C). The transfection efficiency of myc-tagged TRPC3 and Orai1 was confirmed by Western blotting (Figure 4F and Supplemental Figure 5A). Overexpression of TRPC1 also amended ER $\mathrm{Ca}^{2+}$ and restored SOC-mediated $\mathrm{Ca}^{2+}$ entry in $\mathrm{MPP}^{+}$-treated $\mathrm{SH}-\mathrm{SY} 5 \mathrm{Y}$ cells when compared with control GFP-expressing cells treated with $\mathrm{MPP}^{+}$(Figure 4, C and D). In agreement with this finding, TRPC1 overexpression decreased the elevations in GRP78, GRP94, and CHOP that were induced after $\mathrm{MPP}^{+}$treatment, indicating that TRPC1 could prevent prolonged UPR activation (Figure 4E; quantification shown in Supplemental Figure 5, D and F). Phosphorylation of PERK, an important transducer of the UPR, and downstream signaling targets (eIF2 $\alpha$ ) was also increased after $\mathrm{MPP}^{+}$treatment, but decreased in TRPC1-overexpressing cells (Figure 4E). Similarly, prolonged activation of the UPR, which has been shown to activate JNK and leads to cell death (31), was increased in $\mathrm{MPP}^{+}$-treated cells but restored to normal in TRPC1-overexpressing cells.

Although Orai1 overexpression did not increased Tg-induced SOC-mediated $\mathrm{Ca}^{2+}$ entry in SH-SY5Y cells (Figure 4B), we still evaluated its role in regulating ER stress, since it has been also shown to contribute to SOC current in some cells (27-29). As shown in Figure 4F, Orail overexpression did not prevent the $\mathrm{MPP}^{+}$-induced ER stress response (quantification of individual proteins shown in Supplemental Figure 5, G-I). To further confirm that the TRPC1dependent decrease in UPR was mediated by SOC-mediated $\mathrm{Ca}^{2+}$ entry through TRPC1, we overexpressed a TRPC1 pore mutant (TRPC1 pm) in SH-SY5Y cells. Consistent with our previous results (32), overexpression of TRPC1 pm failed to increase Tg-induced SOC currents in SH-SY5Y cells (Figure 4B). Interestingly, SH-SY5Y cells overexpressing TRPC1 $1 \mathrm{pm}$ also failed to inhibit $\mathrm{MPP}^{+}$-induced UPR and did not protect against neurotoxin-induced cell death (Figure 4, G and H; quantification shown in Supplemental Figure $5, \mathrm{~J}-\mathrm{L}$ ). Taken together, these results suggest that $\mathrm{MPP}^{+}$induces ER stress by downregulating the function of TRPC1 and that overexpression of functional TRPC1 is crucial for maintaining ER $\mathrm{Ca}^{2+}$ homeostasis and inhibiting $\mathrm{MPP}^{+}$-induced ER stress response, thereby preventing neurodegeneration.

Modulation of AKT is essential for TRPC1-mediated neuroprotection. To better understand the link between TRPC1 and cell survival, we searched for downstream signaling molecules that could be responsible for TRPC1-mediated protection. Given the already 
A

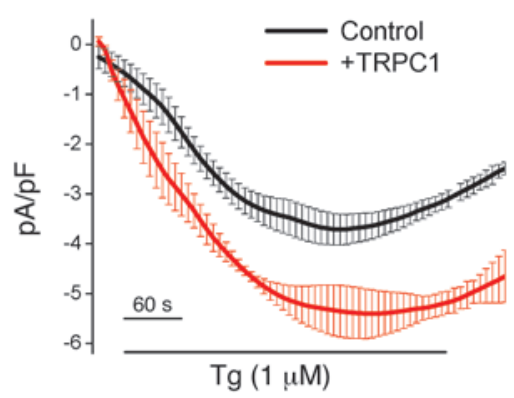

C

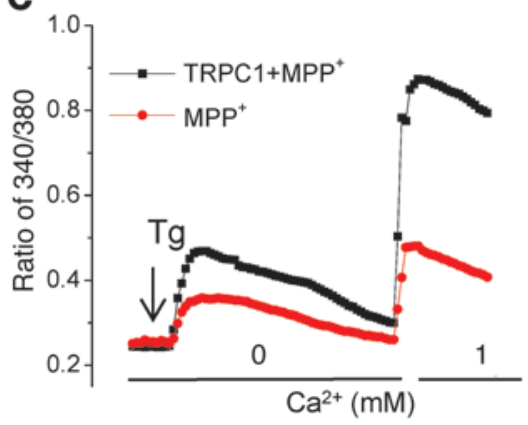

B

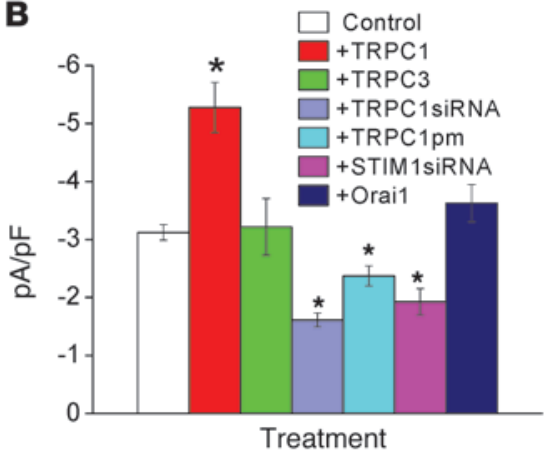

D

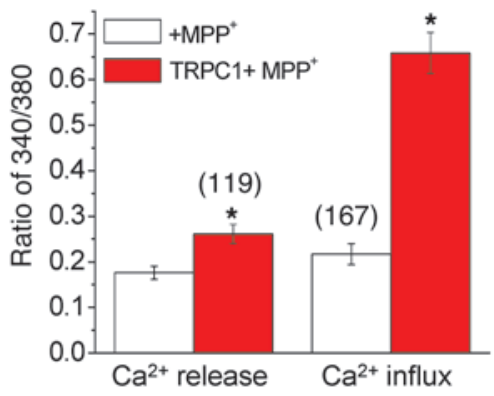

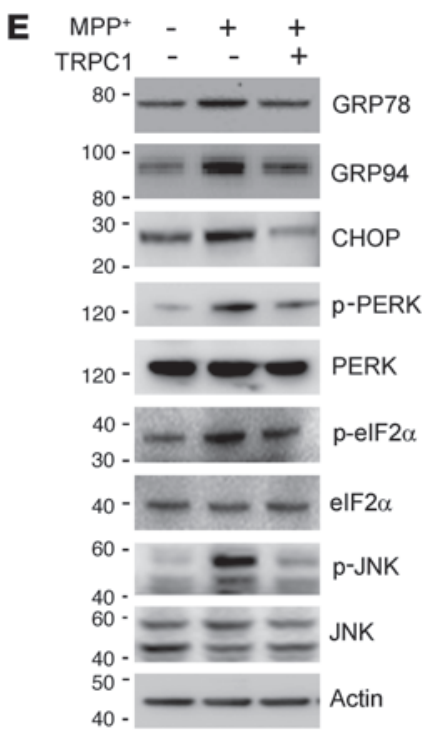

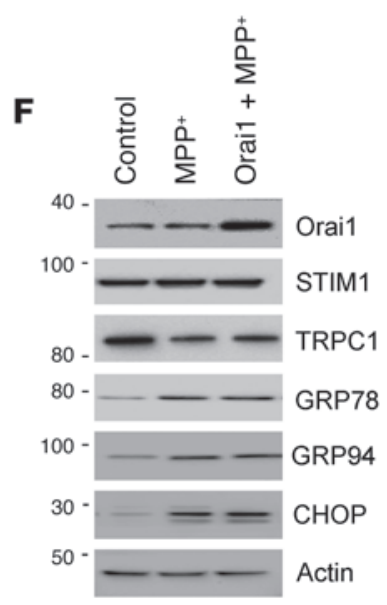

G

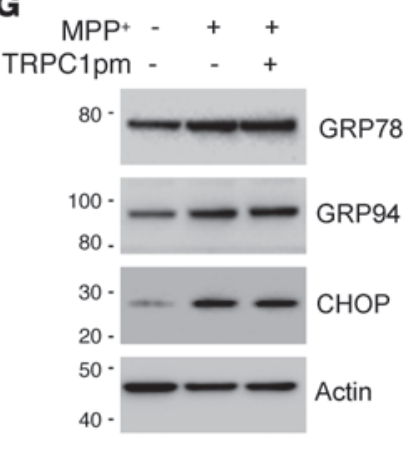

H

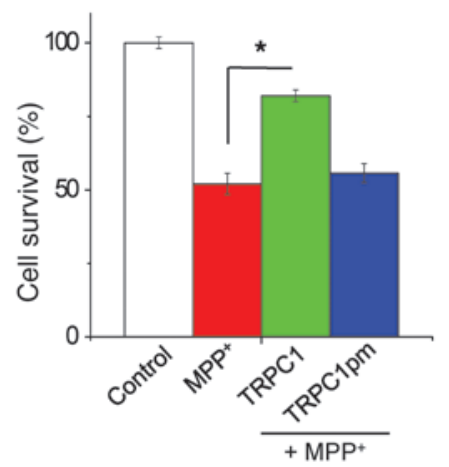

Figure 4

TRPC1 overexpression restores SOCE and attenuates ER stress. (A) Tg-induced currents (at $-80 \mathrm{mV}$, mean \pm SEM) were evaluated in control and TRPC1-overexpressing SH-SY5Y cells. (B) Average (mean \pm SEM from 7-9 recordings) current intensity under various conditions. (C) SHSY5Y cells were treated with MPP+ for 12 hours with or without TRPC1 overexpression, and analog plots (mean \pm SEM) of the fluorescence ratio $(340 / 380 \mathrm{~nm})$ are shown. Fluorescence ratio was measured in the presence of $2 \mu \mathrm{M}$ Tg with and without $1 \mathrm{mM}^{\mathrm{Ca}}{ }^{2+}$. (D) Quantification (mean \pm SEM) of fluorescence ratio; ${ }^{*} P<0.05$ versus MPP+-treated cells. (E-G) TRPC1-, Orai1-, or TRPC1pm-overexpressing SH-SY5Y cells were treated with $\mathrm{MPP}^{+}$for 12 hours, lysed, resolved, and subjected to Western blotting with the indicated antibodies. (H) Cell survival (MTT assay) under different conditions. Values are expressed as mean \pm SD. ${ }^{*} P<0.05$ versus MPP+-treated cells.

known relationship between AKT and neuroprotection (33), we studied whether AKT plays a role in TRPC1-mediated neuroprotection. As shown in Figure 5A, a decrease in AKT phosphorylation was observed in PD patient samples (quantification of individual blots shown in Supplemental Figure 6, A and B). Interestingly, $\mathrm{MPP}^{+}$treatment also significantly decreased AKT1 phosphorylation without affecting total AKT1 levels in $\mathrm{SH}$ SY5Y cells (Figure 5B). In addition, overexpression of full-length TRPC1, but not TRPC1 pm, prevented the decrease in AKT phos- phorylation seen after $\mathrm{MPP}^{+}$treatment (Figure 5, B-D; quantification of phospho-AKT [Ser473] shown in Supplemental Figure 6C). In addition, quantification of the phospho-AKT (Ser473) indicated an approximately $50 \%$ inhibition of the AKT activity after $\mathrm{MPP}^{+}$treatment, which was restored to approximately $75 \%$ in cells overexpressing TRPC1 and treated with $\mathrm{MPP}^{+}$(Figure $5 \mathrm{C})$. We next examined whether SOCE that is dependent on TRPC1 activates AKT phosphorylation in SH-SY5Y cells. Interestingly, SH-SY5Y cells treated with $\operatorname{Tg}(5 \mu \mathrm{M})$ in the absence of 
A

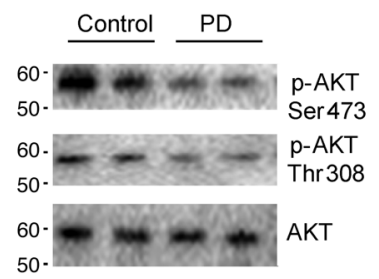

D

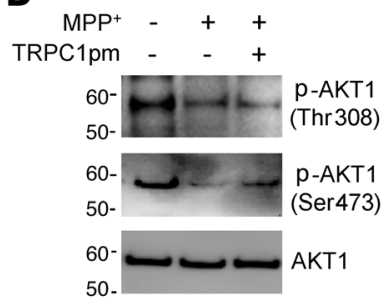

G

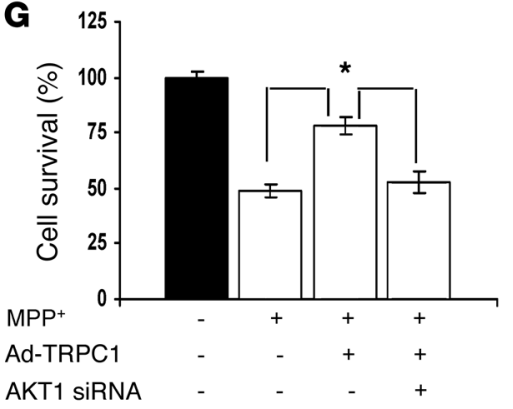

B

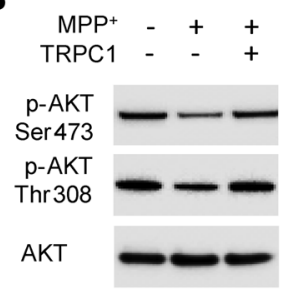

$\mathbf{E}$

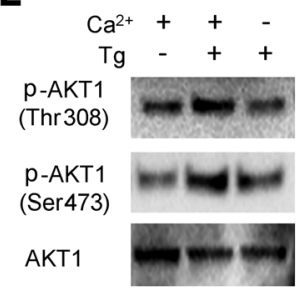

H

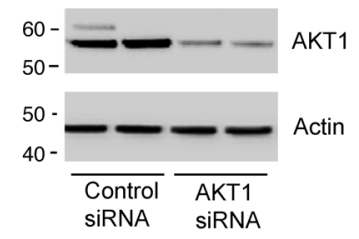

C

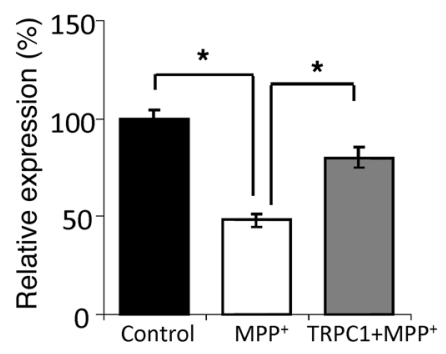

F

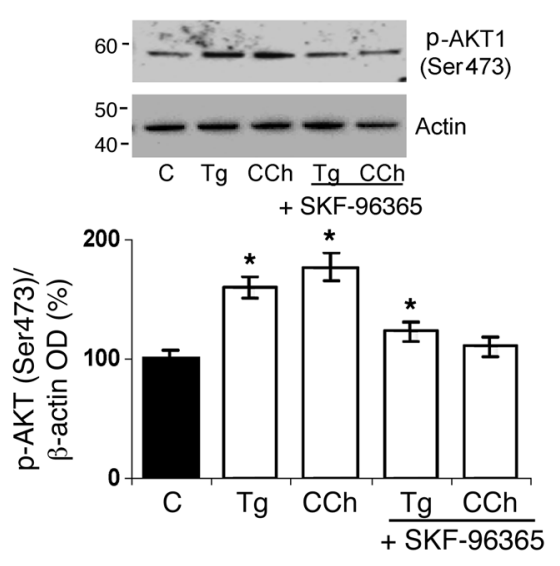

Figure 5

AKT modulation is crucial for TRPC1-mediated neuroprotection. (A) Brain lysates from control and PD samples were immunoblotted with the respective antibodies. (B) Cells were treated with $\mathrm{MPP}^{+}(500 \mu \mathrm{M}, 12$ hours) with or without Ad-TRPC1, and Western blots were performed. For control, membranes were reprobed with anti-AKT1. (C) Relative expression of phospho-AKT1 (Ser473) is shown. Values are mean \pm SD; ${ }^{*} P<0.05$. (D) Cells were transfected with control vector or TRPC1pm (36 hours) and treated with MPP+ $(500 \mu \mathrm{M})$ for 12 hours, lysed, and subjected to Western blotting with the indicated antibodies. (E) SH-SY5Y cells were treated with $5 \mu \mathrm{M} \mathrm{Tg}$ in SES buffer for 10 minutes with or without $\mathrm{Ca}^{2+}(2 \mathrm{mM})$, lysed, resolved using SDS-PAGE, and immunoblotted with AKT1 and phosphorylated AKT antibodies. (F) SH-SY5Y cells were treated with $5 \mu \mathrm{M}$ Tg or $100 \mu \mathrm{M}$ CCh for 15 minutes with or without pretreatment with SKF-96365 (50 $\mu \mathrm{M}, 45$ minutes), processed, and probed with phospho-AKT1 (Ser473). Diagram shows the densitometric values of phospho-AKT1 (Ser473). Values are mean \pm SEM. ${ }^{*} P<0.05$ versus untreated controls. (G) SH-SY5Y cells were transfected with AKT1 siRNA and/or with Ad-TRPC1, followed by the addition of MPP+ (12 hours) and assayed for cell survival. Values represent mean $\pm \mathrm{SD}$ from at least 3 independent experiments. ${ }^{*} P<0.05$ versus untreated control. $(\mathrm{H}) \mathrm{SH}$ SY5Y cells were transfected with 50 pmol AKT1 siRNA, lysed after 36 hours, resolved, and immunoblotted with anti-AKT1 and anti- $\beta$-actin.

external $\mathrm{Ca}^{2+}$ failed to show AKT phosphorylation (Figure 5E), suggesting that $\mathrm{Ca}^{2+}$ influx through SOCs was necessary for AKT1 phosphorylation, as $\mathrm{Ca}^{2+}$ release from internal ER stores by itself was not sufficient to activate AKT1 phosphorylation (quantification shown in Supplemental Figure 6D). Moreover, stimulation of TRPC1 by Tg or carbachol (CCh) (known activators of TRPC1) significantly increased AKT1 phosphorylation (Ser473) when compared with control untreated cells (Figure 5F). Furthermore, addition of SKF-96365 prevented the activation of AKT1 induced by $\mathrm{Tg}$ and CCh (Figure 5F). To evaluate whether other sources of $\mathrm{Ca}^{2+}$ influx can also stimulate AKT phosphorylation, we stimulated SH-SY5Y cells with oleyl-acetyl-glycerol (OAG), which is known to activate other TRPC channels and is independent of store depletion (34). Interestingly, AKT phosphorylation was not altered upon OAG stimulation
(Supplemental Figure 6E), suggesting that the effect observed in AKT phosphorylation is dependent on $\mathrm{Ca}^{2+}$ entry via the SOC channel. In addition, expression of brain-derived neurotrophic factor (BDNF) was also evaluated, since $\mathrm{Ca}^{2+}$ entry is known to induce the expression of these factors, which has been shown to increase protection of DA cells. As indicated in Supplemental Figure 6F, addition of $\mathrm{MPP}^{+}$significantly decreased BDNF expression; however, no increase in BDNF expression was observed in cells overexpressing TRPC1, suggesting that TRPC1mediated protection is independent of BDNF. To further evaluate the role of TRPC1 and AKT in cell survival, we performed MTT assays. $\mathrm{MPP}^{+}$-treated cells showed a significant decrease in neuronal survival, which was inhibited by TRPC1 overexpression (Figure 5G). Additionally, silencing of AKT1 completely blocked TRPC1-mediated neuroprotection against $\mathrm{MPP}^{+}$, indicating that 
A
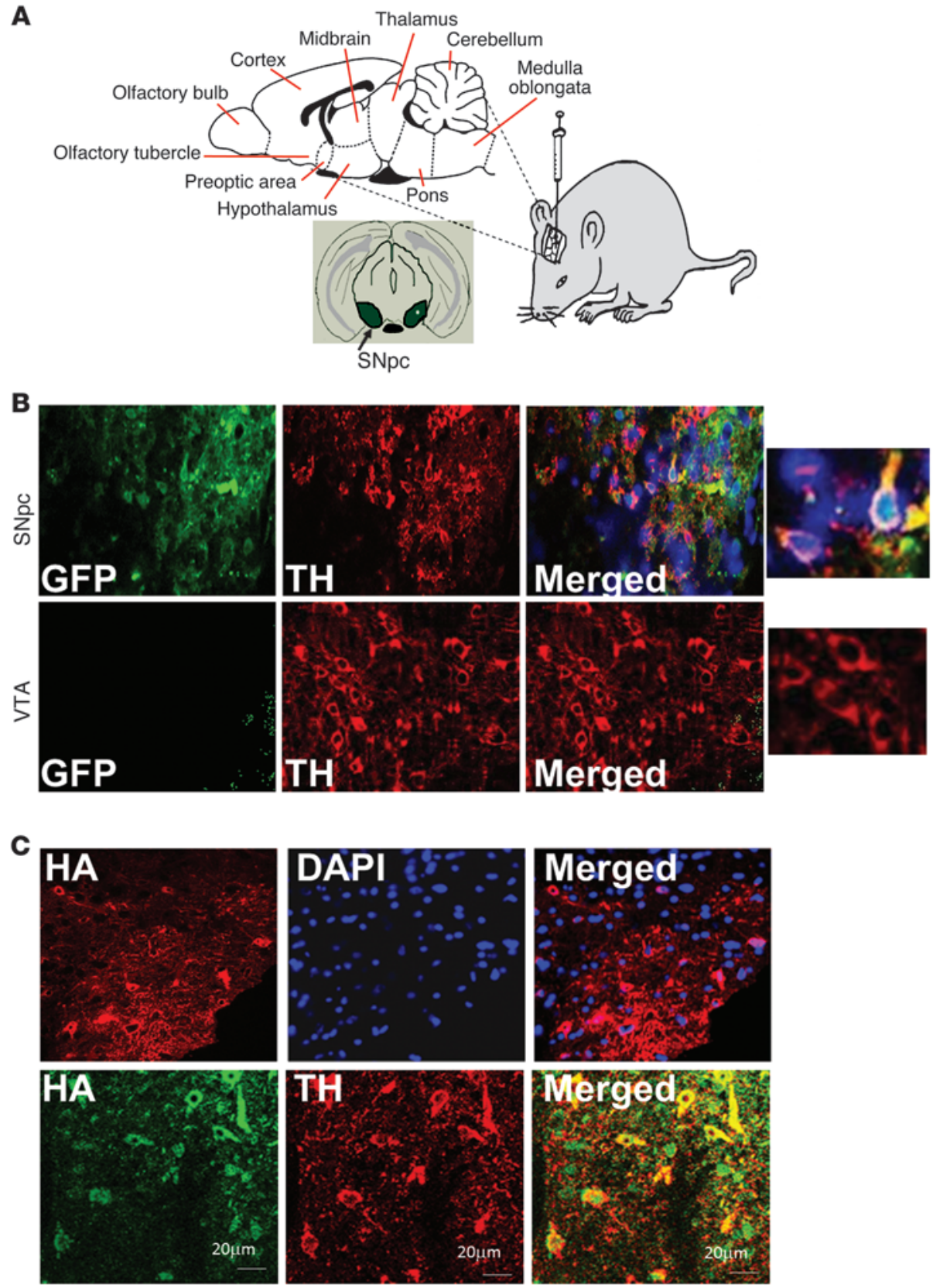
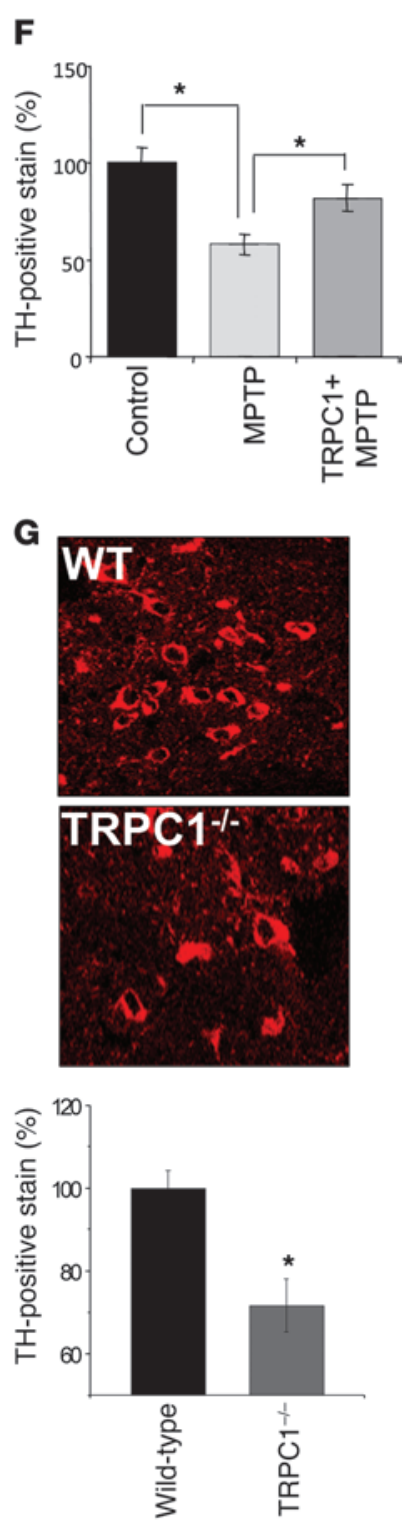

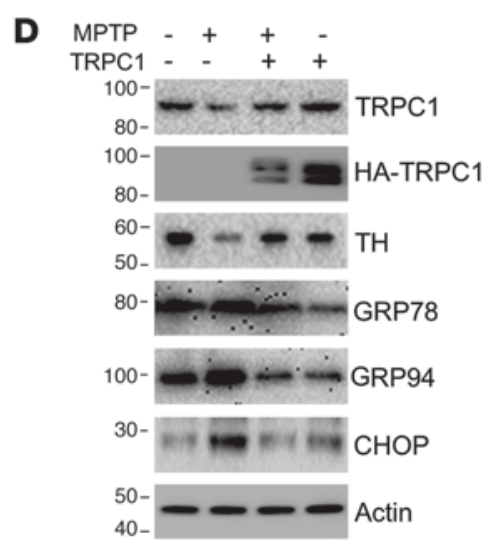

E
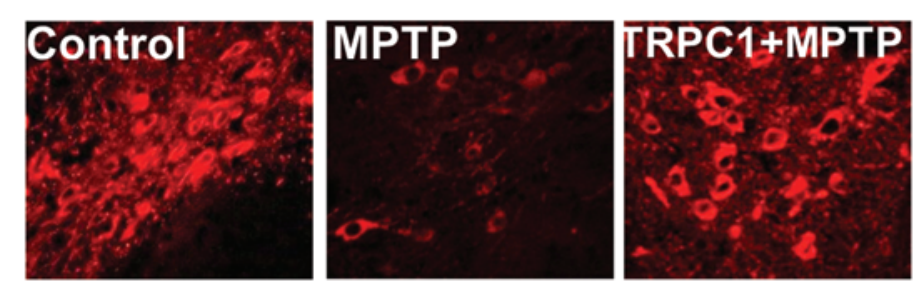


\section{Figure 6}

TRPC1 overexpression protects DA neuron in an in vivo MPTP model of PD. (A) Graphic representation of intranigral injection protocol. (B and $\mathbf{C}$ ) Unilateral injection of GFP or HA-TRPC1 adenovirus $\left(3 \times 10^{7}\right.$ particles) into the SNpc $(n=6)$. Brain samples containing the SNpc region were sectioned $(12 \mu \mathrm{m})$ and stained for $\mathrm{TH}$ immunofluorescence. Expression of GFP and TH in the SNpc and in ventral tegmental area (VTA) were evaluated. HA-TRPC1 colocalized with TH (C, bottom row). Scale bars in C: $20 \mu \mathrm{m}$. (D) HA-TRPC1 or GFP adenovirus was injected directly into the SNpc of animals ( $n=6-8$ per group) 7 days before MPTP treatment. After 1 week of MPTP injection, SNpc samples were removed and subjected to SDSPAGE and immunoblotted with the respective antibodies. Control GFP virus-injected mice received an equal volume of saline. (E) Representative TH staining of the ipsilateral sides of animals injected with the indicated virus with and without MPTP. (F) Quantification of the number of $\mathrm{TH}$-positive neurons from ipsilateral or contralateral sides for the indicated treatment groups. Data are mean \pm SEM. ${ }^{*} P<0.05$. (G) Brain samples containing the SNpc from wild-type and $\operatorname{Trpc1} 1^{-/-}$mice were sectioned $(12 \mu \mathrm{m})$ and stained for TH. Quantification of TH-positive neurons is shown in the graph. Data are presented as mean \pm SEM. ${ }^{\star} P<0.05$. Original magnification, $\times 40$; magnified images in $\mathbf{B}, \times 100$.

AKT1 plays a crucial role in TRPC1-mediated neuroprotection (Figure 5, G and $\mathrm{H}$ ). These results strongly suggest that TRPC1mediated $\mathrm{Ca}^{2+}$ influx is vital for AKT1 activation in SH-SY5Y cells, which is critical for their survival.

TRPC1 overexpression protects DA neurons in an in vivo MPTP model of $P D$. While the above results strongly suggest the importance of TRPC1 in cellular models of PD, nothing is known regarding the role of TRPC1 in an in vivo PD model. Thus, we overexpressed HA-TRPC1 in the SNpc region by intranigral injection of AdTRPC1 as shown in Figure 6A. Control mice received intranigral injection of Ad-GFP, and as indicated in Figure 6B, GFP was expressed in DA neurons of the SNpc and colocalized with tyrosine hydroxylase ( $\mathrm{TH}$, a marker for DA neurons), indicating that we had been successful in targeting the SNpc with our injections. Thus, we next injected Ad-HATRPC1 and confirmed by confocal microscopy the overexpression of TRPC1 (HA-TRPC1), which also colocalized with TH-positive neurons (DA neurons) of SNpc (Figure 6C). Also as expected, MPTP treatment decreased the expression of TH and TRPC1 in SNpc (Figure 6D; quantification of individual proteins shown in Supplemental Figure 7, A and B). Importantly, MPTP treatment induced ER stress in DA neurons by activating the UPR, which was inhibited in mice treated with MPTP but overexpressing TRPC1 (Figure 6D; quantification of individual proteins shown in Supplemental Figure 7, C-E). To further understand the role of TRPC1 in the protection of DA neurons, we evaluated TH staining under these conditions. MPTP induces neuronal degeneration of DA neurons, which was indicated by the decrease in TH levels in MPTP-injected mice (Figure $6 \mathrm{E})$. Importantly, a significant increase in TH-positive neurons was observed in TRPC1-overexpressing mice treated with MPTP. Quantification of the data indicated approximately $80 \%$ survival of DA neurons in TRPC1-overexpressing mice following MPTP treatment (Figure 6F). To further confirm these results, we quantified TH-positive neurons in wild-type and $\operatorname{Trpc1} 1^{-/-}$mice, since the results shown above indicated that $\operatorname{Trpc1} 1^{-/-}$mice have decreased SOC-mediated $\mathrm{Ca}^{2+}$ entry and increased ER stress. A significant decrease in TH-positive neurons was observed in $\operatorname{Trpc1}^{-/-}$mice even without MPTP treatment (Figure 6G).
In vivo TRPC1 overexpression activates the AKT/mTOR pathway. The above results clearly suggest that TRPC1 overexpression prevented prolonged UPR activation and attenuated the degeneration of DA neurons in an in vivo PD model. However, the signaling intermediates linking TRPC1 and DA neuron survival in PD are still unknown. We therefore examined whether in vivo overexpression of TRPC1 would activate the AKT/mTOR pathway. Importantly, MPTP treatment attenuated the activation of mTOR, a kinase that regulates neuronal survival, in SNpc (Figure 7A; quantification shown in Supplemental Figure 7F). This mTOR suppression could in turn suppress its downstream proteins that are involved in cellular signaling. Consistent with our in vitro observations (Figure $5 \mathrm{~B}$ ), as shown in Figure 7B, treatment with MPTP decreased the phosphorylation of AKT at both Ser473 and Thr378 in the SNpc, as indicated by Western blotting. These observations indicate that MPTP impaired the functions of AKT/mTOR in DA neurons and thereby induced neurodegeneration. Interestingly, TRPC1 overexpression in SNpc significantly restored the activation of mTOR and its downstream targets (Figure 7A). Consistent with this, TRPC1 overexpression in SNpc prevented the suppression of AKT1 activation by MPTP (Figure 7B; quantification shown in Supplemental Figure 7, G and H). These results suggest that TRPC 1 is necessary to restore $\mathrm{AKT} / \mathrm{mTOR}$ activation and in the protection of DA neurons.

\section{Discussion}

Disturbances in ER $\mathrm{Ca}^{2+}$ homeostasis have been associated with many neurological disorders including PD $(7,17,35)$. Disruption of $\mathrm{ER} \mathrm{Ca}^{2+}$ homeostasis induces the UPR, which is a pro-survival defense mechanism that prevents further accumulation of newly synthesized proteins in the ER in order to reduce further burden to the ER. However, prolonged UPR activation occurs when physiological mechanisms fail to restore normal ER function, thereby causing ER stress and cell death (10). Thus, disturbances in ER $\mathrm{Ca}^{2+}$ homeostasis could play an important role in neurodegenerative diseases. Our studies provide direct evidence that inhibition of SOCE by $\mathrm{MPP}^{+}$promotes ER $\mathrm{Ca}^{2+}$ depletion during the early phase and that a decrease in TRPC1 function leads to ER stress and subsequent cell death. Importantly, it has been shown that depletion of ER Ca ${ }^{2+}$ stores is toxic to SH-SY5Y cells and that $\mathrm{Ca}^{2+}$ chelators increase cell death $(36,37)$. These studies are consistent with our results and imply that restoration of $\mathrm{ER} \mathrm{Ca}^{2+}$ stores, which depends on TRPC1 activity, can protect SH-SY5Y cells.

$\mathrm{Ca}^{2+}$ release from internal ER stores plays an important role in maintaining normal cell function. $\mathrm{Ca}^{2+}$ entry through SOC channels not only ensures optimal refilling of the ER, but also leads to a prolonged increase in cytosolic $\mathrm{Ca}^{2+}$. Importantly, both TRPC and Orai channels have been shown to mediate $\mathrm{Ca}^{2+}$ entry upon store depletion (22-29). Our results indicate that although other TRPCs and Orais are expressed in DA cells/neurons, MPTP $/ \mathrm{MPP}^{+}$specifically targets TRPC1. Furthermore, the endogenous SOC has I-V relationships that are similar to those observed for TRPC1-dependent currents (26). Importantly, SOC-mediated $\mathrm{Ca}^{2+}$ entry decreased 2- to 3-fold in $\mathrm{MPP}^{+}$-treated cells, and since only TRPC1 expression was decreased, we infer that the loss of endogenous SOC-mediated $\mathrm{Ca}^{2+}$ entry was due to the loss of TRPC1. Our results offer a mechanism by which $\mathrm{MPP}^{+}$induces ER stress, which is consistent with previous reports that addition of $\mathrm{MPP}^{+}$causes ER stress $(16,17,38)$. Consistent with this, Brandman et al. have shown that basal SOC-mediated $\mathrm{Ca}^{2+}$ entry maintains $\mathrm{ER} \mathrm{Ca}^{2+}$ homeostasis and that a decrease in SOC-mediat- 

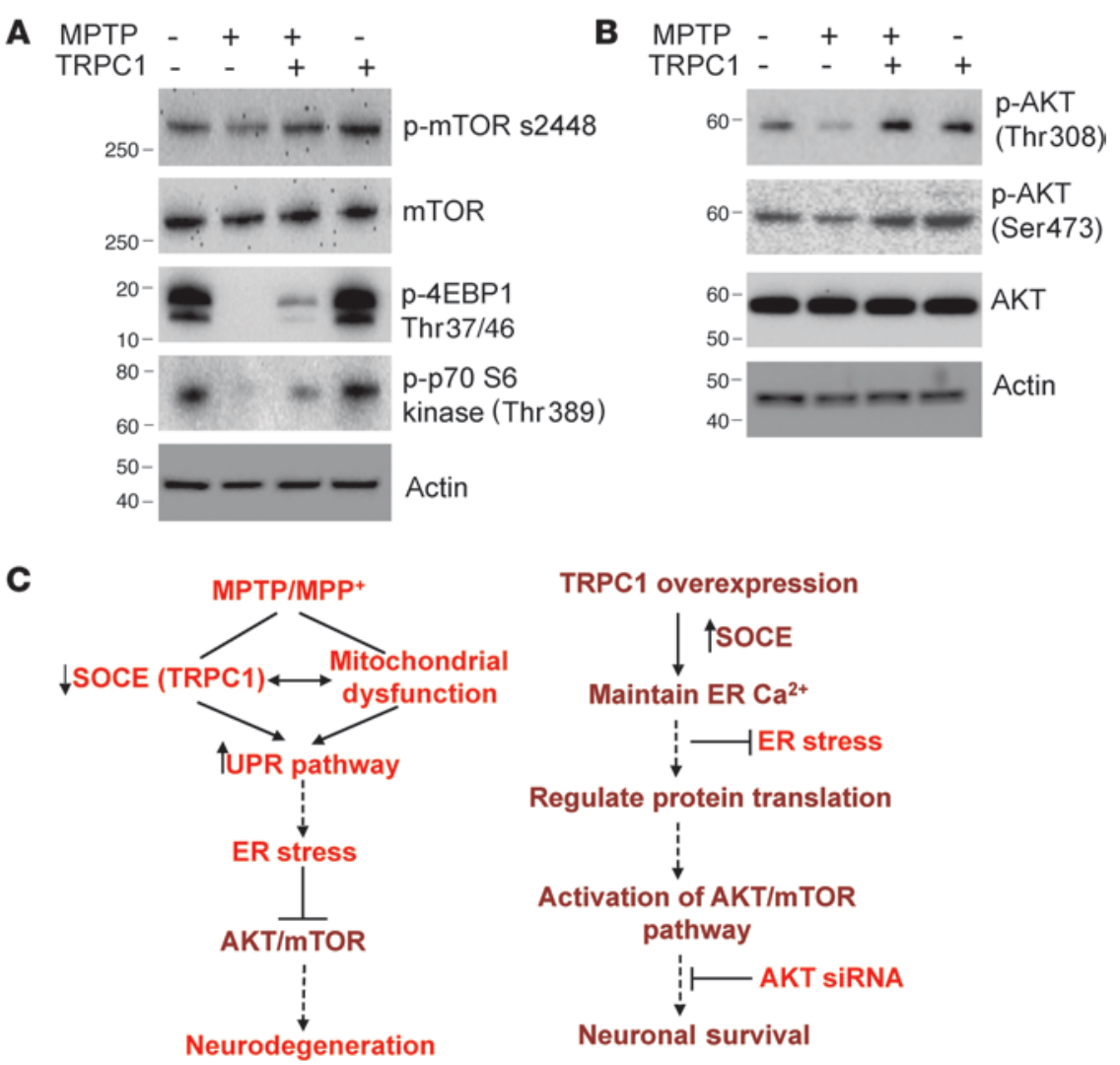

\section{Figure 7}

TRPC1 overexpression activates the AKT/ mTOR pathway in mice. (A and B) SNpc regions were removed from control animals and animals overexpressing TRPC1 that had been treated or not with MPTP, and were subjected to SDSPAGE and immunoblotting with the respective antibodies. Data are representative of 2-3 independent experiments. (C) Model for MPP+/ MPTP-induced DA loss and TRPC1-mediated neuroprotection. MPP+/MPTP decreases the expression of TRPC1 and SOC-mediated $\mathrm{Ca}^{2+}$ influx either directly or indirectly via mitochondrial dysfunction. This leads to prolonged ER $\mathrm{Ca}^{2+}$ depletion and activation of the UPR and subsequent ER stress-mediated neurodegeneration. In contrast, TRPC1 overexpression restores SOCE function and maintains ER $\mathrm{Ca}^{2+}$ homeostasis. Further, $\mathrm{Ca}^{2+}$ influx through TRPC1 activates AKT/mTOR-mediated survival mechanisms in DA cells, which leads to increased neuronal survival. ed $\mathrm{Ca}^{2+}$ entry contributes to the reduction in $\mathrm{ER} \mathrm{Ca}^{2+}$ content (39). Importantly, TRPC1 silencing also caused decreased $\mathrm{ER} \mathrm{Ca}^{2+}$ and $\mathrm{Ca}^{2+}$ influx, suggesting that TRPC1 mediates SOC-mediated $\mathrm{Ca}^{2+}$ entry in $\mathrm{SH}-\mathrm{SY} 5 \mathrm{Y}$ cells. However, it is still unclear how MPTP/MPP ${ }^{+}$impairs TRPC1 channel activity. One possibility is that $\mathrm{MPP}^{+}$could induce mitochondrial membrane depolarization, which could contribute to the reduction in SOC-mediated $\mathrm{Ca}^{2+}$ entry, since mitochondria have a fundamental role in regulating this type of $\mathrm{Ca}^{2+}$ entry $(40,41)$. The other possibility is that $\mathrm{MPP}^{+}$could directly inhibit TRPC1 channel activity; more research is needed to explore this concept.

Various physiological conditions that are known to be associated with ER stress have been shown to alter ER $\mathrm{Ca}^{2+}$ homeostasis (37). We observed that inhibition of SOC-mediated $\mathrm{Ca}^{2+}$ entry by $\mathrm{MPP}^{+}$leads to a decrease in $\mathrm{ER} \mathrm{Ca}^{2+}$, which in turn induces ER stress. Our data substantiate recent studies indicating that $\mathrm{MPP}^{+}$ induces ER stress through a mechanism involving the depletion of ER $\mathrm{Ca}^{2+}$ (38). Importantly, blocking TRPC channel activity or TRPC1 silencing, but not TRPC3 silencing, activates the UPR pathway. Consistent with these results, the UPR markers were significantly increased in the midbrain region of $\operatorname{Trpc1}^{-/-}$mice, and there was a significant decrease in SOC-mediated $\mathrm{Ca}^{2+}$ entry and $\mathrm{TH}$-positive neurons. These results are important, since they highlight for the first time to our knowledge that either TRPC1 silencing or inhibition of TRPC channel activity activates ER stress by altering SOC-mediated $\mathrm{Ca}^{2+}$ entry, which contributes to a decrease in $\mathrm{ER} \mathrm{Ca}^{2+}$. We further suggest that the $\mathrm{MPP}^{+}$-induced $\mathrm{ER} \mathrm{Ca}^{2+}$ depletion is directly influenced by TRPC1-mediated changes in $\mathrm{Ca}^{2+}$ entry. Moreover, silencing of STIM1 also activated the UPR in SH-SY5Y cells. STIM1 is an ER $\mathrm{Ca}^{2+}$ binding protein that senses $\mathrm{ER} \mathrm{Ca}^{2+}$ levels, and upon store depletion, STIM1 aggregates and interacts with TRPC1 and Orai1 channels, thereby activating SOCmediated $\mathrm{Ca}^{2+}$ entry $(42,43)$. Interestingly, STIM1 has also been shown to inactivate voltage-gated channels, and $\mathrm{Ca}^{2+}$ entry via the voltage-gated channels has been shown to be deleterious for DA neurons (44-46). Thus, it is possible that activation of TRPC1 via its interaction with STIM1 could inhibit voltage-gated channels and thereby protect DA neurons.

The documentation of the significance of TRPC1 in neuroprotection against store depletion-induced ER stress by MPTP/ $\mathrm{MPP}^{+}$is, to our knowledge, a novel aspect of this study, as it lends credence to previous studies pointing to a neuroprotective role of TRPC channels in the SNpc. $\mathrm{Ca}^{2+}$ influx through TRPC channels appears to be a critical component of the signaling cascade that mediates growth cone guidance and survival of neurons in response to several growth factors $(47,48)$. In particular, recent studies have shed light on the neuroprotective effect of TRPC channels in the SNpc against Tat neurotoxicity (49). Our previous studies also show the neuroprotective action of TRPC1 against in vitro cell culture models of PD (19); however, the precise mechanisms by which TRPC1 regulates neuronal survival remained poorly understood. In this study, we showed that TRPC1 overexpression confers protection against ER stress in both in vivo and in vitro models of PD. TRPC1 overexpression, but not a TRPC1 pore mutant that has decreased permeability to $\mathrm{Ca}^{2+}$, prevented $\mathrm{MPP}^{+}$-mediated cell death by inhibiting the elevation of CHOP and JNK. Also, in our studies TRPC1-mediated neuroprotection against ER stress-induced neurodegeneration correlated with its ability to maintain ER $\mathrm{Ca}^{2+}$ homeostasis, since the TRPC1 pore mutant failed to rescue SH-SY5Y cells from $\mathrm{MPP}^{+}$-induced ER stress and cell death. 
Our findings also unravel the downstream signaling pathways through which TRPC1 promotes neuronal survival induced by a neurotoxin that mimics PD. We observed that $\mathrm{MPP}^{+}$decreases AKT1 activation by decreasing cellular levels of phosphorylated AKT1, which is consistent with previous studies indicating that $\mathrm{PD}$-inducing neurotoxins including $\mathrm{MPP}^{+}$and 6-OHDA decrease phospho-AKT $(50,51)$. Interestingly, TRPC1 overexpression prevented MPTP $/ \mathrm{MPP}^{+}$-mediated loss of AKT1 function by increasing its phosphorylation. AKT1 plays a major role in neuronal survival by phosphorylating its substrates, including BAD, GSK3, NF- $\mathrm{B}$, and forkhead proteins (52-55), and AKT1 overexpression has been shown to protect against $\mathrm{MPP}^{+}$(33). TRPC1 overexpression activates the phosphorylation of AKT at both Ser473 and Thr308, which are necessary for complete activation of AKT1. Also, $\mathrm{Ca}^{2+}$ influx via TRPC1 was necessary for the activation of AKT1, since removal of external $\mathrm{Ca}^{2+}$ prevented, whereas addition of external $\mathrm{Ca}^{2+}$ restored, AKT1 phosphorylation. Similarly, the TRPC1pm was unable to activate AKT1 phosphorylation in $\mathrm{MPP}^{+}$-treated cells. These findings were further confirmed by the use of pharmacological TRPC channel activators (Tg and CCh) and its inhibitor (SKF96365). Activation of TRPC1 by Tg and CCh led to increased phospho-AKT1 (Ser473), whereas pretreatment with SKF-96365 significantly prevented TRPC1-mediated AKT1 phosphorylation. More importantly, TRPC1 exerted neuroprotection via AKT activation, since silencing AKT1 abolished TRPC1-mediated neuroprotection in SH-SY5Y cells. Although no increase in BDNF expression was observed in TRPC1-overexpressing cells treated with $\mathrm{MPP}^{+}$, we cannot rule out the possibility that the release of BDNF under these conditions is also not altered.

Consistent with the in vitro studies, we found that overexpression of TRPC1 in the mouse SNpc also resulted in rescue of MPTP-mediated loss of DA neurons. We previously reported that MPTP treatment decreases the membrane expression of TRPC1. Consistent with this, the present study also showed that MPTP treatment significantly decreased TRPC1 expression and increased activation of UPR markers in the SNpc. Growing evidence also indicates the importance of the mTOR pathway in apoptosis and autophagy that can lead to neuronal death, but in all these cases it was the inhibition of the AKT phosphorylation, rather than mTOR activation, that eventually led to neuronal loss $(51,56)$. Our results show that MPTP represses the phosphorylation of AKT, mTOR, p70 S6 kinase, and 4EBP1 and that loss of AKT leads to neuronal loss. Importantly, mTOR kinases are downstream of the AKT pathway and have been shown to have a dual role (in both neuronal survival and neuronal demise); however, it is the activation of the AKT pathway that might phosphorylate mTOR differently that can have a positive effect rather than leading to neuronal loss, as observed in some of these studies. ER stress induced by tunicamycin has shown to downregulate the activity of AKT and mTOR and induced apoptosis in rat hippocampal neurons (55). In contrast, Deguil et al. reported that there were no significant differences in the expression of $\mathrm{mTOR}$ and its downstream protein in the midbrain of MPTP-treated mice, though the changes were observed in the striatum, frontal cortex, and hippocampus (57). Interestingly, our data demonstrate that TRPC1 overexpression protects DA neurons by preventing MPTP-induced ER stress, which is evidenced by increased survival of TH-positive DA neurons in mice that overexpressed TRPC1 and were treated with MPTP.
To relate these observations to human disease, we used postmortem SNpc samples from PD and non-PD individuals. Our results indicate that TRPC1 expression is decreased in the SNPc of PD patients, and activation of UPR proteins is increased. Consistent with previous studies, the level of AKT phosphorylation was also decreased in the SNpc of samples from PD patients (56), and since our cellular models indicate that loss of TRPC1 due to $\mathrm{MPP}^{+} / \mathrm{MPTP}$ treatment decreases AKT phosphorylation, it could be anticipated that loss of AKT activation in PD samples is due to the loss of TRPC1. Overall, these data support our hypothesis that TRPC1 plays a vital role in maintaining $\mathrm{ER} \mathrm{Ca}^{2+}$ homeostasis and that reduction in its function leads to prolonged activation of the UPR pathway and impairs AKT activation, which subsequently leads to neurodegeneration as observed in PD (Figure 7C).

\section{Methods}

Reagents. $\mathrm{MPP}^{+}$and MPTP were obtained from Sigma-Aldrich. Tg, tunicamycin, and Fura-2 were obtained from Calbiochem. Antibodies that were used in this study are described in Supplemental Table 1. All other reagents used were of molecular biology grade and obtained from Sigma-Aldrich.

Cell culture and transfections. SH-SY5Y cells were obtained from ATCC and cultured/maintained at $37^{\circ} \mathrm{C}$ as previously described (18). SH-SY5Y cells were differentiated by the addition of retinoic acid for 6 days and used for the experiments. $\mathrm{MPP}^{+}$was added to cells and was present during the duration of the experiment (12 hours) unless otherwise stated. For adenoviral expression, SH-SY5Y cells were infected with Ad-TRPC1 at an MOI of 5. For transient transfection, SH-SY5Y cells $\left(1.5 \times 10^{5}\right.$ cells $\left./ \mathrm{ml}\right)$ were transfected with TRPC1 siRNAs (Ambion) or STIM1 siRNA (58) or scrambled control siRNA (Ambion negative control siRNA \#1) using HiPerFect transfection reagent (QIAGEN). Cells were passaged and transfected with siRNA every 3 days when the cells were $80 \%-90 \%$ confluent and in log growth phase. The transfection efficiency of FAM-labeled negative control siRNA (Ambion) was greater than $90 \%$. AKT1 siRNA and control siRNA, obtained from Santa Cruz Biotechnology Inc., were transfected using siRNA transfection reagent (Santa Cruz Biotechnology Inc.) as per the manufacturer's instruction and were used 48 hours after transfection. Cell viability was measured by using the Vybrant MTT cell proliferation assay kit (Molecular Probes, Invitrogen). Absorbance was read at $570 \mathrm{~nm}$ (630 nm as a reference) on a microplate reader (Molecular Devices). Cell viability was expressed as a percentage of the control culture. Lipofectamine 2000 (Invitrogen) was used to transfect myc-Orai1, myc-TRPC3 (Origene Technologies), and TRPC1 pm (32).

Quantitative RT-PCR. TRPC, GRP78, and CHOP mRNA expression was assessed with real-time RT-PCR using commercially available primers (Origene Technologies). cDNA was transcribed from $1 \mu \mathrm{g}$ of total RNA with iScript cDNA (Bio-Rad). An equal amount of cDNA template was added to iQ SYBR Green Supermix together with appropriate primers at $0.2 \mu \mathrm{M}$ each. Quantitative PCR was performed using an iCycler iQ real-time detection system following the specifications of the manufacturer. The relative level of mRNA was interpolated from a standard curve prepared by serially diluting the cDNA reaction. GAPDH was used for normalization of the transcripts. Specificity of PCR product formation was confirmed by monitoring melting peaks.

Immunoprecipitation and Western blotting. Immunoprecipitations were carried out as described earlier $(58,59)$. Following stimulation, cells were lysed with RIPA buffer and used for immunoprecipitation. Proteins were resolved in $4 \%-12 \%$ NuPAGE gels, followed by Western blotting with the desired antibodies. Crude membrane or lysates were prepared from $\mathrm{SH}$ SY5Y cells and from animal and human tissues as previously described $(18,19)$. Protein concentrations were determined using the Bradford 
reagent (Bio-Rad), and 25-50 $\mu \mathrm{g}$ of lysates were resolved on NuPAGE $4 \%-12 \%$ Bis-Tris gel or NuPAGE 3\%-8\% Tris-acetate gels (Invitrogen), followed by Western blotting as described in refs. 18, 19 .

Calcium measurements and electrophysiology. SH-SY5Y cells were incubated with $2 \mu \mathrm{M}$ Fura-2 (Molecular Probes, Invitrogen) for 45 minutes and washed twice with $\mathrm{Ca}^{2+}$-free SES buffer as described in ref. 19. For patch clamp experiments, coverslips with cells were transferred to the recording chamber and perfused with an external Ringer's solution of the following composition (in $\mathrm{mM}$ ): $\mathrm{NaCl}, 145 ; \mathrm{KCl}, 5 ; \mathrm{MgCl}_{2}, 1 ; \mathrm{CaCl}_{2}, 1 ; \mathrm{HEPES}, 10$; glucose, $10 ; \mathrm{pH} 7.4(\mathrm{NaOH})$. The divalent-free solution (DVF) contained (in mM) $165 \mathrm{NaCl}, 5 \mathrm{CsCl}, 10$ EDTA, 10 HEPES, and 10 glucose, $\mathrm{pH}$ 7.4. The patch pipette had resistances between 3 and $5 \mathrm{~m} \Omega$ after being filled with the standard intracellular solution containing the following (in $\mathrm{mM}$ ): cesium methane sulfonate, $145 ; \mathrm{NaCl}, 8$; $\mathrm{MgCl}_{2}, 10$; HEPES, 10; EGTA, 10; pH 7.2 (CsOH). All electrophysiological experiments were performed using a previously described protocol (26). The maximum peak currents were calculated at a holding potential of $-80 \mathrm{mV}$. The I-V curves were made using a ramp protocol, whereby current density was evaluated at various membrane potentials and plotted. For the tabulation of statistics, peak currents were used as described in ref. 26.

Brain slice preparation and DA cell identification. Fifteen- to 22-day-old mice were sacrificed, and brain was dissected out in ice-cold saline solution. Coronal brain sections $(400 \mu \mathrm{m})$ were cut using a vibrating blade microtome (VT1000S; Leica). Neurons were visualized with infrared videomicroscopy (Olympus BX51WI) and differential interference contrast optics. Recording electrodes were filled with (in mM) $110 \mathrm{CsCl}, 5 \mathrm{MgCl}_{2}, 10$ EGTA, 10 HEPES, 4 Na-ATP, and 0.1 GTP, pH 7.4. The extracellular solution comprised (in mM) $130 \mathrm{NaCl}, 24 \mathrm{NaHCO}_{3}, 3.5 \mathrm{KCl}, 1.25 \mathrm{NaH}_{2} \mathrm{PO}_{4}, 2.5 \mathrm{CaCl}_{2}$, $1.5 \mathrm{MgCl}_{2}, 0.25$ TTX, and 10 glucose, saturated with $95 \% \mathrm{O}_{2}$ and $5 \% \mathrm{CO}_{2}$, $\mathrm{pH} 7.4$ Data were filtered at $2 \mathrm{kHz}$, digitized at $10 \mathrm{kHz}$, and acquired and analyzed using PCLAMP 10 software (Molecular Devices). The DA neurons differ from GABA neurons based on their electrophysiological properties, which included hyperpolarization-activated inward current $\left(I_{b}\right)$. To evoke an $I_{b}$ current, a hyperpolarizing pulse of $60 \mathrm{mV}$ and of 1.5 -second duration was applied to all cells. An $I_{b}$ current ratio was calculated by measuring the current at the end of the capacitive transient over the amplitude of the current at the end of the voltage command. For DA neurons, $I_{b}$ is pronounced $\left(I_{\mathrm{h}}\right.$ ratio $\left.<0.6\right)$, whereas for GABA neurons $I_{b}$ is small $\left(I_{b}\right.$ ratio $\left.>0.6\right)$.

Luciferase reporter assays. SH-SY5Y cells were transfected with either $100 \mathrm{ng}$ of ERSE reporter (SABiosciences) or negative/positive controls using Lipofectamine 2000 in Opti-MEM (Invitrogen). After 4 hours of transfection, cells were infected with adenovirus expressing HA-TRPC1 in DMEM/F12. Cells were then incubated for 36 hours before induction. For the $\mathrm{MPP}^{+}$induction, fresh medium with $500 \mu \mathrm{M} \mathrm{MPP}^{+}$was added to each well. After $\mathrm{MPP}^{+}$treatment, cells were lysed, and a dual luciferase assay was performed following the manufacturer's instructions (Promega). Luciferase activity was measured using a Zylux Femtomaster FB12 luminometer (PerkinElmer).

Immunofluorescence. Animals were anesthetized and perfused with PBS, followed by paraformaldehyde ( $4 \%, \mathrm{w} / \mathrm{v})$ in PBS. The brain was removed intact and postfixed overnight in paraformaldehyde, cryoprotected in $30 \%$ sucrose in PBS for $24-48$ hours at $4^{\circ} \mathrm{C}$, and then frozen in O.C.T. freezing compound (Ted Pella Inc.). Serial cryosections were collected through the entire midbrain $(12 \mu \mathrm{M})$. All samples were examined and images obtained using a Zeiss Meta confocal microscope. For quantitative measurements, researchers blind to the treatment protocol counted the TH-positive neurons in the SNpc. Measurements from 4-6 sections per brain were averaged to obtain one value per subject.

Animals. Eight- to 10-month-old male $\operatorname{Trpc1} 1^{-/}$and wild- type mice were used for this experiment. The generation of $\operatorname{Trpc1} 1^{-/-}$mice was described previously (26). All animals were housed in a temperature-controlled room under a 12-hour light/12-hour dark cycle with ad libitum access to food and water. All animal experiments were carried out according to University of North Dakota guidelines for the use and care of animals.

Injection into the substantia nigra. Mice received unilateral injection of either control virus (Ad-GFP) or Ad-HA-TRPC1 $\left(3 \times 10^{7}\right.$ particles in $\left.3 \mu \mathrm{l}\right)$ into the substantia nigra (coordinates from bregma: anterior-posterior, -3.3 ; mediallateral, -1.5 ; dorsal-ventral, -4.6$)$. The solution was injected into the substantia nigra with a $10 \mu \mathrm{l} \mathrm{Hamilton} \mathrm{syringe} \mathrm{coupled} \mathrm{to} \mathrm{a} \mathrm{motorized} \mathrm{injector}$ (Stoelting) at a rate of $0.3 \mu \mathrm{l} / \mathrm{min}$, and the needle was left in place for at least 10 minutes after injection. After 1 week of adenovirus injection, mice were challenged with MPTP (MPTP-HCl, $25 \mathrm{mg} / \mathrm{kg}$ per injection, i.p.) for 5 consecutive days at 24-hour intervals as described in ref. 19. Mice were sacrificed 7 days after the last MPTP injection, and the brain was removed from the skull and placed with the dorsal side up. Using a scalpel blade, a coronal cut was made adjacent to the inferior colliculi approximately at bregma $-6.36 \mathrm{~mm}$. A second cut was made approximately at bregma -2.54 $\mathrm{mm}$, based on the mouse brain atlas. The ventral midbrain was dissected to ensure that there was no contamination of the hippocampus, cortex, or cerebellum. Brain regions from 2-3 animals were pooled for each experiment.

Human brain samples. Frozen and paraffin-embedded blocks of postmortem human substantia nigral samples of control and PD patients (stage 3-4 based on the Hoehn and Yahr scale; ref. 60) were obtained from the UK Parkinson's Disease Society Tissue Bank at Imperial College and the Udall Center at the University of Pennsylvania (Philadelphia, Pennsylvania, USA). The frozen tissues were used to isolate RNA and proteins, and expression of genes and proteins was evaluated using RT-PCR and Western blotting as described above. Immunofluorescence was performed on $8-\mu \mathrm{m}$ sections using TRPC1 and TH antibodies as described above.

Statistics. Data analysis was performed using Origin 7.0 (OriginLab). Statistical comparisons were made using Student's $t$ test (2-tailed). Experimental values are expressed as mean $\pm \mathrm{SD}$ or mean $\pm \mathrm{SEM}$. Differences in the mean values were considered to be significant at $P<0.05$.

Study approval. The study protocols were approved by the Institutional Review Board and Institutional Animal Care and Use Committee of the University of North Dakota. Informed consent was not required, since we used autopsy samples donated to the brain bank.

\section{Acknowledgments}

We greatly acknowledge the use of the Edward C. Carlson Imaging and Image Analysis Core Facility. We especially thank Laura Leiphon and Virginia Achen for their help. We greatly acknowledge the UK Parkinson's Disease Society Tissue Bank at Imperial College and John Trojanowski (University of Pennsylvania) for providing the PD and control samples. Also, grant support from the National Science Foundation (no. 0548733) and the NIH (DE017102 and 5P20RR017699) awarded to B.B Singh; from the NIH Intramural Program (project Z01-ES-101684) to L. Birnbaumer; and from the National Institute of Neurological Disorders and Stroke (NINDS; P50 NS-053488) awarded to John Trojanowski is acknowledged.

Received for publication October 5, 2011, and accepted in revised form January 11, 2012.

Address correspondence to: Brij B Singh or Senthil Selvaraj, Department of Biochemistry and Molecular Biology, School of Medicine and Health Sciences, University of North Dakota, $501 \mathrm{~N}$ Columbia Road, Grand Forks, North Dakota 58201, USA. Phone: 701.777.0834; Fax: 701.777.2382; E-mail: brij.singh@med.und.edu (B.B Singh). Phone: 701.777.2012; Fax 701.777.2382; E-mail: senthilbio@gmail.com (S. Selvaraj). 
1. Dauer W, Przedborski S. Parkinson's disease: mechanisms and models. Neuron. 2003;39(6):889-909.

2. Marras C, Lang A. changing concepts in Parkinson disease: moving beyond the decade of the brain. Neurology. 2008;70(21):1996-2003.

3. Abou-Sleiman PM, Muqit MM, Wood NW. Expanding insights of mitochondrial dysfunction in Parkinson's disease. Nat Rev Neurosci. 2006;7(3):207-219.

4. Chan CS, et al. 'Rejuvenation' protects neurons in mouse models of Parkinson's disease. Nature. 2007;447(7148):1081-1086

5. Bezprozvanny I. Calcium signaling and neurodegenerative diseases. Trends Mol Med. 2009;15(3):89-100.

6. Balch WE, Morimoto RI, Dillin A, Kelly JW. Adapting proteostasis for disease intervention. Science. 2008;319(5865):916-919.

7. Paschen W, MengesdorfT. Endoplasmic reticulum stress response and neurodegeneration. Cell Calcium. 2005;38(3-4):409-415.

8. Hiller MM, Finger A, Schweiger M, Wolf DH. ER degradation of a misfolded luminal protein by the cytosolic ubiquitin-proteasome pathway. Science. 1996;273(5282):1725-1728.

9. Boyce M, Yuan J. Cellular response to endoplasmic reticulum stress: a matter of life or death. Cell Death Differ. 2006;13(3):363-373.

10. Harding HP, Calfon M, Urano F, Novoa I, Ron D. Transcriptional and translational control in the Mammalian unfolded protein response. Annu Rev Cell Dev Biol. 2002;18:575-599.

11. Chiba K, Trevor A, Castagnoli N Jr. Metabolism of the neurotoxic tertiary amine, MPTP, by brain monoamine oxidase. Biochem Biophys Res Commun. 1984;120(2):574-578

12. Przedborski S, Tieu K, Perier C, Vila M. MPTP as a mitochondrial neurotoxic model of Parkinson's disease. J Bioenerg Biomembr. 2004;36(4):375-379.

13. Kitahama K, Denney RM, Maeda T, Jouvet M. Distribution of type B monoamine oxidase immunoreactivity in the cat brain with reference to enzyme histochemistry. NeuroScience. 1991;44(1):185-204.

14. Lotharius J, Dugan LL, O'Malley KL. Distinct mechanisms underlie neurotoxin-mediated cell death in cultured dopaminergic neurons. J Neurosi. 1999;19(4):1284-1293

15. Javitch JA, D’Amato RJ, Strittmatter SM, Snyder $\mathrm{SH}$. Parkinsonism-inducing neurotoxin, $\mathrm{N}$-methyl4-phenyl-1,2,3,6-tetrahydropyridine: uptake of the metabolite $\mathrm{N}$-methyl-4-phenylpyridine by dopamine neurons explains selective toxicity. Proc Nat Acad Sci U S A. 1985;82(7):2173-2177.

16. Holtz WA, O’Malley KL. Parkinsonian mimetics induce aspects of unfolded protein response in death of dopaminergic neurons. $J$ Biol Chem. 2003;278(21):19367-19377.

17. Hoozemans JJ, van Haastert ES, Eikelenboom P, de Vos RA, Rozemuller JM, Scheper W. Activation of the unfolded protein response in Parkinson's disease. Biochem Biophys Res Commun. 2007;354(3):707-711.

18. Bollimuntha S, Singh BB, Shavali S, Sharma SK, Ebadi M. TRPC1-mediated inhibition of 1methyl-4-phenylpyridinium ion neurotoxicity in human SH-SY5Y neuroblastoma cells. J Biol Chem. 2005;280(3):2132-2140.

19. Selvaraj S, Watt JA, Singh BB. TRPC1 inhibits apoptotic cell degeneration induced by dopaminergic neurotoxin MPTP/MPP(+). Cell Calcium. 2009;46(3):209-218.

20. Montell C. Physiology, phylogeny, and functions of the TRP superfamily of cation channels. Sci STKE. 2001;2001(90):re1.
21. Liao Y, Erxleben C, Yildirim E, Abramowitz J, Armstrong DL, Birnbaumer L. Orai proteins interact with TRPC channels and confer responsiveness to store depletion. Proc Natl Acad Sci U S A. 2007;104(11):4682-4687

22. Birnbaumer L. The TRPC class of ion channels: a critical review of their roles in slow, sustained increases in intracellular $\mathrm{Ca}^{2+}$ concentrations. Annu Rev Pharmacol Toxicol. 2009;49:395-426.

23. Putney JW. Physiological mechanisms of TRPC activation. Pflugers Arch. 2005;451(1):29-34.

24. Cahalan MD. STIMulating store-operated $\mathrm{Ca}(2+)$ entry. Nat Cell Biol. 2009;11(6):669-677.

25. Parekh AB, Penner R. Store depletion and calcium influx. Physiol Rev. 1997;77(4):901-930.

26. Liu X, et al. Attenuation of store-operated Ca2+ current impairs salivary gland fluid secretion in TRPC1(-/-) mice. Proc Natl Acad Sci U S A. 2007; 104(44):17542-17547

27. Yuan JP, Zeng W, Dorwart MR, Choi YJ, Worley PF, Muallem S. SOAR and the polybasic STIM1 domains gate and regulate Orai channels. Nat Cell Biol. 2009;11(3):337-343.

28. Wang Y, Deng X, Gill DL. Calcium signaling by STIM and Orai: intimate coupling details revealed. SciSignal. 2010;3(148):pe42.

29. Liao Y, Plummer NW, George MD, Abramowitz J, Zhu MX, Birnbaumer L. A role for Orai in TRPC-mediated $\mathrm{Ca} 2+$ entry suggests that a TRPC: Orai complex may mediate store and receptor operated $\mathrm{Ca}^{2+}$ entry. Proc Natl Acad Sci U S A. 2009;106(9):3202-3206.

30. Riccio A, et al. mRNA distribution analysis of human TRPC family in CNS and peripheral tissues. Brain Res Mol Brain Res. 2002;109(1-2):95-104.

31. Liu CY, Kaufman RJ. The unfolded protein response. J Cell Sci. 2003;116(pt 10):1861-1862.

32. Liu X, Singh BB, Ambudkar IS. TRPC1 is required for functional store-operated $\mathrm{Ca}^{2+}$ channels. J Biol Chem. 2003;278(13):11337-11343.

33. Salinas M, Martin D, Alvarez A, Cuadrado A. Akt1/ PKBalpha protects PC12 cells against the parkinsonism-inducing neurotoxin 1-methyl-4-phenylpyridinium and reduces the levels of oxygen-free radicals. Mol Cell Neurosci. 2001;17(1):67-77.

34. Hofmann T, Obukhov AG, Schaefer M, Harteneck C, Gudermann T, Schultz G. Direct activation of human TRPC6 and TRPC3 channels by diacylglycerol. Nature. 1999;397(6716):259-263.

35. Mattson MP. ER calcium and Alzheimer's disease: in a state of flux. Sci Signal. 2010;3(114):pe10.

36. Nguyen HN, Wang C, Perry DC. Depletion of intracellular calcium stores is toxic to SH-SY5Y neuronal cells. Brain Res. 2002;924(2):159-166

37. Rizzuto R, et al. Close contacts with the endoplasmic reticulum as determinants of mitochondrial $\mathrm{Ca}^{2+}$ responses. Science. 1998;280(5370):1763-1766.

38. Chigurupati $\mathrm{S}$, et al. The homocysteine-inducible endoplasmic reticulum stress protein counteracts calcium store depletion and induction of CCAAT enhancer-binding protein homologous protein in a neurotoxin model of Parkinson disease. J Biol Chem. 2009;284(27):18323-18333.

39. Brandman O, Liou J, Park WS, Meyer T. STIM2 is a feedback regulator that stabilizes basal cytosolic and endoplasmic reticulum $\mathrm{Ca}^{2+}$ levels. Cell. 2007;131(7):1327-1339.

40. Glitsch MD, Bakowski D, Parekh AB. Store-operated $\mathrm{Ca}^{2+}$ entry depends on mitochondrial $\mathrm{Ca} 2+$ uptake. EMBO J. 2002;21(24):6744-6754.

41. Hoth M, Button DC, Lewis RS. Mitochondrial control of calcium-channel gating: a mechanism for sustained signaling and transcriptional activation in T lymphocytes. Proc Natl Acad Sci U S A. 2000;97(19):10607-10612

42. Liou J, et al. STIM Is a $\mathrm{Ca}^{2+}$ sensor essential for $\mathrm{Ca}^{2+}$-store-depletion-triggered $\mathrm{Ca}^{2+}$ influx. Curr Biol. 2005;15(13):1235-1241.

43. Lewis RS. The molecular choreography of a store-operated calcium channel. Nature. 2007; 446(7133):284-287

44. Park CY, Shcheglovitov A, Dolmetsch R. The CRAC channel activator STIM1 binds and inhibits L-type voltage-gated calcium channels. Science. 2010;330(6000):101-105

45. Wang Y, et al. The calcium store sensor, STIM1, reciprocally controls Orai and CaV1.2 channels. Science. 2010;330(6000):105-109.

46. Chan CS, et al. Rejuvenation' protects neurons in mouse models of Parkinson's disease. Nature. 2007;447(7148):1081-1086.

47. Wang GX, Poo MM. Requirement of TRPC channels in netrin-1-induced chemotropic turning of nerve growth cones. Nature. 2005;434(7035):898-904.

48. Talavera K, Nilius B, Voets T. Neuronal TRP channels: thermometers, pathfinders and life-savers. Trends Neurosci. 2008;31(6):287-295.

49. Yao $\mathrm{H}$, et al. Involvement of TRPC channels in CCL2-mediated neuroprotection against tat toxicity. J Neurosci. 2009;29(6):1657-1669.

50. Inoue $\mathrm{H}$, et al. Inhibition of the leucine-rich repeat protein LINGO-1 enhances survival, structure, and function of dopaminergic neurons in Parkinson's disease models. Proc Natl Acad Sci U S A. 2007;104(36):14430-14435.

51. Malagelada C, Jin ZH, Jackson-Lewis V, Przedborski $S$, Greene LA. Rapamycin protects against neuron death in in vitro and in vivo models of Parkinson's disease. J Neurosci. 2010;30(3):1166-1175.

52. Cross DA, Alessi DR, Cohen P, Andjelkovich M, Hemmings BA. Inhibition of glycogen synthase kinase- 3 by insulin mediated by protein kinase B. Nature. 1995;378(6559):785-789.

53. Brunet A, Datta SR, Greenberg ME. Transcriptiondependent and -independent control of neuronal survival by the PI3K-Akt signaling pathway. Curr Opin Neurobiol. 2001;11(3):297-305.

54. del Peso L, Gonzalez-Garcia M, Page C, Herrera R, Nunez G. Interleukin-3-induced phosphorylation of BAD through the protein kinase Akt. Science. 1997;278(5338):687-689.

55. Di Nardo A, et al. Tuberous sclerosis complex activity is required to control neuronal stress responses in an mTOR-dependent manner. J Neurosci. 2009;29(18):5926-5937.

56. Malagelada C, Jin ZH, Greene LA. RTP801 is induced in Parkinson's disease and mediates neuron death by inhibiting Akt phosphorylation /activation. J Neurosci. 2008;28(53):14363-14371.

57. Deguil J, Chavant F, Lafay-Chebassier C, PéraultPochat MC, Fauconneau B, Pain S. Neuroprotective effect of PACAP on translational control alteration and cognitive decline in MPTP parkinsonian mice. Neurotox Res. 2010;17(2):142-155.

58. Pani B, et al. Activation of TRPC1 by STIM1 in ER$\mathrm{PM}$ microdomains involves release of the channel from its scaffold caveolin-1. Proc Natl Acad Sci US A. 2009;106(47):20087-20092.

59. Ong HL, et al. Dynamic assembly of TRPC1-STIM1Orai1 ternary complex is involved in store-operated calcium influx. J Biol Chem. 2007;282(12):9105-9116.

60. Hoehn M, Yahr M. Parkinsonism: onset, progression and mortality. Neurology. 1967;17(5):427-442. 